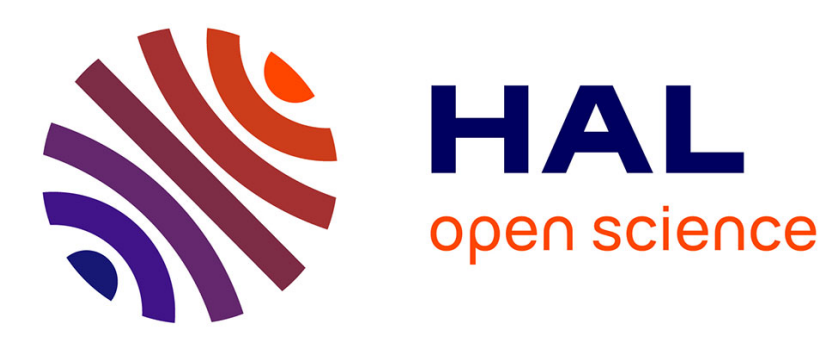

\title{
Toward Luminescent Iron Complexes: Unravelling the Photophysics by Computing Potential Energy Surfaces
}

Antonio Francés-monerris, Philippe Gros, Xavier Assfeld, Antonio Monari, Mariachiara Pastore

\section{- To cite this version:}

Antonio Francés-monerris, Philippe Gros, Xavier Assfeld, Antonio Monari, Mariachiara Pastore. Toward Luminescent Iron Complexes: Unravelling the Photophysics by Computing Potential Energy Surfaces. ChemPhotoChem, 2019, 3 (9), pp.666-683. 10.1002/cptc.201900100 . hal-03445128

\section{HAL Id: hal-03445128 \\ https://hal.science/hal-03445128}

Submitted on 23 Nov 2021

HAL is a multi-disciplinary open access archive for the deposit and dissemination of scientific research documents, whether they are published or not. The documents may come from teaching and research institutions in France or abroad, or from public or private research centers.
L'archive ouverte pluridisciplinaire HAL, est destinée au dépôt et à la diffusion de documents scientifiques de niveau recherche, publiés ou non, émanant des établissements d'enseignement et de recherche français ou étrangers, des laboratoires publics ou privés. 


\title{
Toward luminescent iron complexes: unraveling the photophysics by computing potential energy surfaces
}

\author{
Antonio Francés-Monerris, ${ }^{*[a]}$ Philippe C. Gros, ${ }^{[b]}$ Xavier Assfeld, ${ }^{[a]}$ Antonio Monari, ${ }^{[a]}$ and Mariachiara \\ Pastore $^{*,[a]}$
}

\begin{abstract}
Due to its high societal impact, the replacement of precious metals used in technological devices by more abundant and ecofriendly metals like iron has stimulated many scientific efforts in the last years. In the present review, we focus on different computational strategies and techniques used to characterize the potential energy surfaces (PESs) that govern the photophysical paths of a wide variety of $\mathrm{Fe}$ (II) complexes. The different procedures are discussed in terms of accuracy, computational cost and availability of the implementations, and illustrated with specific examples taken from the literature. The determination of minimum energy paths (MEPs) determinations and the optimization of minimum energy crossing points (MECPs) are particularly emphasized since they can be combined to provide connected and optimized PESs independent from any a priori selected coordinate. The use of such computational techniques is exemplified in detail through a recent study on the influence of the facial and meridional isomerism in the triplet PESs of a pyridylcarbene $\mathrm{Fe}(\mathrm{II})$ complex, and its implications in the decay mechanism of each isomer.
\end{abstract}

\section{Introduction}

Transition metal complexes (TMCs) possessing sufficiently longlived metal to ligand charge transfer (MLCT) excited states play a crucial role in a plethora of relevant physico-chemical processes at the base of different technological applications, spanning from natural and artificial photosynthesis, ${ }^{[1-4]}$ photovoltaic application $\mathrm{s}^{[5-8]}$ to light-assisted medical therapies. ${ }^{[9-11]}$ Since the first paper in $1960 \mathrm{~s}^{[12]}$ on the luminescence of $\left.[\mathrm{Ru}(\mathrm{bpy})]^{2+}\right]^{2+}$ and the following work by Gafney and Adamson, ${ }^{[13]}$ in which the electron transfer (ET) quenching of the triplet charge transfer (CT) excited state was demonstrated, ruthenium polypyridyl complexes have dominated the scene in photovoltaics and solar fuels devices. ${ }^{[14-}$ 20] One of the reasons behind the unsurpassed success of $\mathrm{Ru}(\mathrm{II})$ based photosensitizers and photocatalysts can be ascribed to their high electron injection quantum yields ${ }^{[1,21-24]}$ originating from the fast electron injection rates compared to the intrinsically long lifetimes (from tens to hundreds of nanoseconds) of their MLCT lowest excited states.

[a] Dr. A. Francés-Monerris, Prof. X. Assfeld, Dr. A. Monari, Dr. M. Pastore

Laboratoire de Physique et Chimie Théoriques (LPCT) Université de Lorraine, CNRS, 54000 Nancy (France)

E-mail: antonio.frances@univ-lorraine.fr, mariachiara.pastore@univlorraine.fr

[b] Dr. P. C. Gros

Laboratoire Lorrain de Chimie Moléculaire (L2CM)

Université de Lorraine, CNRS, 54000 Nancy (France)
The study of the photophysics of TMCs has greatly benefitted from the outstanding development of time-resolved spectroscopy techniques, allowing a femtosecond resolution of the different relaxation processes, and covering not only the UV/Vis and IR domain but also $\mathrm{X}$-ray wavelengths, hence also providing information on the structural reorganization ${ }^{[25-28]}$ associated to the photoresponses, crucial to characterize the photophysics of TMCs.

Despite its record efficiencies and the fulfilment of all desired photophysical criteria, ruthenium suffers from serious drawbacks, which potentially limit its large-scale applicability, mainly related to its toxicity and rarity. To this respect, a straightforward solution would be the use of TMCs based on Earth's abundant first row metals, ${ }^{[29]}$ such as iron, which is largely more copious, cheaper and environmentally friendly. However, the ${ }^{1,3} \mathrm{MLCT}$ states in conventional $\mathrm{Fe}$ (II)-polypyridyl complexes undergo ultrafast (ca. $100 \mathrm{fs}$ ) deactivation to low-lying metal-centered (MC) states, via the triplet ${ }^{3} \mathrm{MC}$ and ultimately the quintuplet ${ }^{5} \mathrm{~T}_{2}$ states, ${ }^{[30-35]}$ thus impeding any efficient utilization of photoinduced electron transfer reactions. ${ }^{[36-38]}$ The reason, as depicted in Figure 1, lies in the reversed energy order of MLCT and MC states, that can be in turn traced back to fundamental electronic structure characteristics of the first-row metals. ${ }^{[39]}$ The absence of a radial nodal plane at a large distance from the nucleus for $n=3$ and $l=2$ (3d) wavefunctions causes a less effective screening of the nuclear charge and thus a "contraction" of the $3 d$ orbitals. ${ }^{[40]}$ Such contraction reduces the orbital overlap between the metal and the ligands and, as a consequence, the ligand-field strength. This inherent weaker ligand-field splitting in $\mathrm{Fe}(\mathrm{II})$-polypyridyl octahedral complexes, compared to the $\mathrm{Ru}(\mathrm{II})$ analogous, induces a stabilization of the MC states in the formers, whereas the MLCT states, whose energy is related to the metal oxidation potential, remains essentially unaffected. This explains the inversion of states shown in Figure 1, and the opening of fast deactivation channels to low energy MC states in iron complexes.
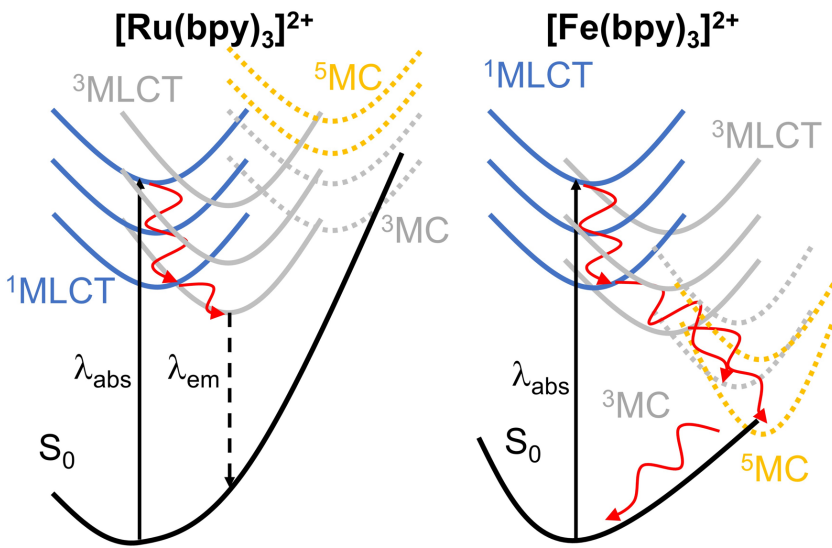
Figure 1. Schematic representation of the radiative excited-state decay of $\left[\mathrm{Ru}(\mathrm{bpy})_{3}\right]^{2+}$ and the non-radiative photoresponse of $\left[\mathrm{Fe}(\mathrm{bpy})_{3}\right]^{2+}$. Solid black arrows indicate light absorption $\left(\lambda_{\text {abs }}\right)$, dashed black arrows stand for light emission $\left(\lambda_{\mathrm{em}}\right)$, and red curly arrows indicate vibrational relaxation and nonadiabatic population transfer events. MLCT surfaces are represented with solid lines and MC surfaces are displayed as dotted lines.

Various strategies have been exploited in the literature to extend the MLCT lifetime, ${ }^{[41-45]}$ either by increasing the ligand field exploiting structura ${ }^{[46-49]}$ or electronic ${ }^{[49-59]}$ effects, or by further decreasing the ligand field to achieve a high-spin ground state $\left({ }^{5} \mathrm{MC}\right)$ and that can subsequently be photoexcited to a long lived ${ }^{5} \mathrm{MLCT}$ state. ${ }^{[60]}$ The complexation of $\mathrm{Fe}(\mathrm{II})$ with $\mathrm{N}$-heterocyclic carbene (NHC) ligands have represented a breakthrough in the field, extending the MLCT lifetimes by several orders of magnitude..$^{[52,61-64]}$

Even though simple considerations based on the ligand field ${ }^{[65]}$ strength have been successfully exploited to increase the MLCT states lifetime of iron-based TMCs, as recently reviewed in detail by Wenger, ${ }^{[29,41]}$ an increasing number of experimental and theoretical works ${ }^{[55-57,59,66-74]}$ has highlighted the importance of acquiring a more refined understanding of the different electronic, structural and environmental effects governing the excited states relaxation pathways. Quite general and comprehensive reviews concerning the application of computational tools to get insights into the excited states deactivation mechanisms in TMCs has been recently reported by Sousa et a ${ }^{[75]}$ and Escudero, ${ }^{[76]}$ while a more specific discussion about the spin-crossover modelling in iron coordination compounds can be found in the thorough revision conducted by Ashley and Jakubikova. ${ }^{[73]}$ Thus, here we will just shortly summarize the main computational and methodological issues concerning the accurate prediction of the ground and excited states electronic properties in TMCs in complex environments, mainly focusing our discussion, in the next sections, on the different available computational techniques to study potential energy surfaces (PESs).

\subsection{Electronic structure methods for Fe(II) complexes}

Undoubtedly, the bedrock of any computational approach aimed at getting an accurate estimate of the system's photophysics is the calculation of the correct relative energies of the ground and excites states involved in the deactivation pathway. A reliable prediction of both vertical (Franck-Condon region) and adiabatic energy differences is mandatory to figure out the excitation process and the evolution of the active excited states, and this possibly requires reliable geometry optimizations and frequency calculations for both ground and excited states taking into account different spin multiplicities (low vs. high spin configurations).

\subsubsection{Density functional theory}

Due to the considerably large systems size and the possibility to easily include (bulk) solvation effects, ${ }^{[77,78]}$ the method of choice for electronic structure calculations on TMCs has been density functional theory (DFT) and its time dependent extension (TDDFT). As a matter of fact, one of the most debated and benchmarked issue is the ability of various DFT functionals in correctly predicting the relative energetics of high and low spin states. ${ }^{[50,52,53,79-93]}$ The energy difference between high and low spin states was found to be sensitive to the amount of HartreeFock exchange included in the exchange-correlation functional, ${ }^{[82]}$ with the high spin configuration generally overstabilized by the increase of non-local exchange. Although one of the most popular and largely applied functionals for the calculation of different spin states energetics is the B3LYP* (Hartree-Fock exchange reduced to $15 \%),{ }^{[82]}$ other functionals have been successfully employed, with overall accuracies strongly dependent on the system under consideration. Analogously, various functionals have been tested for the TDDFT excited states calculations, showing once again strongly system-dependent results. ${ }^{[50,52,53,94-103]}$ Even though a full treatment of the problem of TDDFT accuracy would be far beyond the scope of the present review, the use of long-range corrected functionals may allow tackling the issue of the correct representation of charge-transfer states in TMCs. The interested reader may be referred to the review recently published by Bokarev et al. ${ }^{[104]}$ Extensive benchmarks of DFT against wavefunction-based approaches on both spin state energetics and optical properties have also been reported in the literature. [105-110] On the other hand, there is consensus regarding the use of the Tamm-Dancoff approximation to avoid triplet instabilities and allow reliable singlet-triplet comparisons. ${ }^{[75]}$

\subsubsection{Ab initio multiconfigurational methods}

As is common for photophysical and photochemical processes, among the most widely employed ab initio methodologies, we quote the complete-active-space self-consistent field (CASSCF)/multireference second-order perturbation theory (MRPT2) approaches, such as the CASPT2 ${ }^{[111]}$ and NEVPT2 ${ }^{[112]}$ methods, that are particularly suited to treat strongly correlated open shell systems and electronically excited states. ${ }^{[68,113-127]}$ In this kind of approaches, the choice of an adequate active space (in terms of size and "physical relevance") is mandatory and can dramatically alter the reliability of the perturbative results as the system's size increases. For this reason, the computational chemist should perform a systematic assessment of the CASSCF zero-order results against active spaces of different sizes to ensure the validity of the reference wave function. ${ }^{[128]}$ Depending on the system, various combination of occupied and virtual orbitals have been employed, like the scheme proposed by Pierloot and Vancoillie, ${ }^{[105]}$ that includes the metal $3 \mathrm{~d}$ orbitals, two $\sigma$ occupied ligand orbitals along the metal-ligand bond, three $\pi^{*}$ virtual orbitals localized over the ligand, and the second $4 \mathrm{~d}$ shell of the iron centre. Moreover, as discussed in Ref. ${ }^{[15]}$, the main limitation in the application of MRPT2 approaches to TMCs, characterized by a high-density of low-lying quasi-degenerated states having a strong multiconfigurational nature and a different CT character, consists on the use of a state-averaged CASSCF procedure. Indeed, to preserve a reasonable accuracy at the perturbative level, the number of states included in the stateaverage CASSCF should be limited to a maximum of 15-20. Furthermore, the states having a remarkably different nature with respect to the rest of the state-averaged manifold, such as the ground state, will be poorly described and hence prone to be overcorrected by the subsequent perturbation step. 


\subsection{Computational approaches to study the solvent effects}

Another point deserving some additional comments concerns the effects of the environment (solvent) on the electronic and optical properties and its computational treatment. For TMCs, the standard TDDFT/PCM ${ }^{[77,78]}$ protocol, employing pure or hybrid functionals with a moderate fraction of HF exchange performs satisfactorily. ${ }^{[129][130]}$ On the other hand, when dealing with CT electronic transitions, one can expect that the surrounding solvent responding to the sudden change in the solute's charge density, plays an important role influencing both the energetics and dynamics of the excited states. ${ }^{[71,131-134]}$ From a computational and theoretical point of view, a proper modelling of the environment as well as of its reorganization to the change in the electronic distribution is crucial for a reliable prediction of any photochemical and photophysical process. This evidently requires to explicitly consider solvent molecules, resorting to $a b$ initio $^{[135]}$ or classical ${ }^{[136]}$ molecular dynamic (MD) simulations; hybrid quantum mechanics/molecular mechanics (QM/MM) schemes, where the solute is described at QM level and the solvent molecules by means of classical mechanics, also represent a valuable strategy. ${ }^{[133,137,138]}$ In particular, solventdependent analysis of the transient electronic absorption spectra, ${ }^{[132]}$ supported by computational analysis, ${ }^{[71,133,137,139]}$ suggests a complex role of the solvent. Indeed, the environment should be considered as a main actor in the modification of the solute's excited state charge distribution, often through the formation or breaking of strongly interacting solute-solvent adducts. These results indicate that to get a reliable picture of the ground and excited state charge distribution and its temporal evolution, the local electrostatics and explicit solvent-solute interactions have to be accurately accounted for.

\section{Potential energy surfaces}

Whereas achieving an accurate enough description of the electronic states is a prerequisite to study the photophysical decay of $\mathrm{Fe}$ (II) complexes, the next step is equally important and involves both determining the molecular coordinates that drive the photoresponse and tracking the excited-state nature and energetics along these coordinates. Actually, both tasks are strongly interdependent, since poor descriptions of the relevant excited states may lead to biased deactivation pathways and vice versa, also depending on the description of the deactivating coordinates. To avoid these possible problems, appropriate benchmarks of the chosen electronic structure method (ideally against experimental data) and the employment of more or less sophisticated algorithms to map the surfaces of the excited states of interest are usually recommended. Even though the implementation and availability of these tools are sometimes limited and computationally expensive, especially for the excited state manifold, recent important developments in a variety of broadly used quantum-chemistry packages pave the way to the accurately determination of the relevant PESs.
As a reminder, we recall that PESs arise from the use of the socalled Born-Oppenheimer approximation to solve the Schrödinger equation's eigenvalue problem (eq 1 ):

$$
\widehat{H}_{s} \Psi(\boldsymbol{r}, \boldsymbol{R})=\boldsymbol{E} \boldsymbol{\Psi}(\boldsymbol{r}, \boldsymbol{R})
$$

where $\widehat{\boldsymbol{H}}_{\boldsymbol{s}}$ represents the time-independent (static) Hamiltonian, $\boldsymbol{\Psi}(\boldsymbol{r}, \boldsymbol{R})$ is the total wave function as a function of the electronic $(\boldsymbol{r})$ and nuclear $(\boldsymbol{R})$ coordinates, and $\boldsymbol{E}$ is the total energy of the system. To decouple the nuclear and electronic motions, the total wave function is broken down into its nuclear $\boldsymbol{\psi}_{n}(R)$ and electronic $\boldsymbol{\psi}_{\boldsymbol{e}}(\boldsymbol{r} ; \boldsymbol{R})$ parts (eq 2$)$ :

$$
\Psi(r, R)=\psi_{n}(R) \times \psi_{e}(r ; R)
$$

where $\boldsymbol{\psi}_{\boldsymbol{e}}(\boldsymbol{r} ; \boldsymbol{R})$ depends only parametrically on the nuclear coordinates $\boldsymbol{R}$. The Born-Oppenheimer approximation relies on the fact that atomic nuclei are much heavier than electrons and therefore the latter move much faster than the former. By neglecting the motion of the nuclei, the term $\psi_{n}(r, R)$ can be factorized to allow the solution of eq 1 . Within this approximation, the multielectronic wavefunction describes the electrons moving in the field generated by the motionless nuclei. Thus, subsequent changes in the nuclear coordinates $\boldsymbol{R}$ followed by re-evaluations of the total energy $\boldsymbol{E}$ give rise to the molecular PESs.

\subsection{Excited-state optimizations}

The first step in the characterization of PESs is the optimization of the excited states of interest to obtain the corresponding equilibrium geometries and the adiabatic energy differences. In $\mathrm{Fe}$ (II) complexes, experimental and theoretical studies have repeatedly demonstrated the major relevance of the lowest-lying ${ }^{1,3} \mathrm{MLCT}$ and ${ }^{1,3,5} \mathrm{MC}$ states, even though the specific competition between them and the possible role of higher excited states have to be evaluated in each system, as depicted in section 3.2. In most cases, due to the large size and the high number of electrons, geometry optimizations are only affordable by means of DFT and TD-DFT methods.

Stationary points are characterized by the null value of the electronic gradients with respect to the internal coordinates $\boldsymbol{q}$ (eq 3):

$$
\frac{\partial E}{\partial q_{1}}=\frac{\partial E}{\partial q_{2}}=\cdots=0
$$

The internal coordinates may be combined to yield normal modes, i.e. the coordinates diagonalizing the Hessian matrices and whose eigenvalues are directly related to the harmonic vibrational frequencies $\left(v_{i}\right)$. Minima are defined when all eigenvalues of the Hessian are greater than zero (eq 4):

$$
v_{1}, v_{2} \ldots>0
$$

In terms of structural distortions, the photoexcitation process is conveniently described as a vertical transition happening at the ground-state equilibrium geometry (Franck-Condon region), i.e. 
changes in the molecular geometry induced by photon absorption are neglected. However, extension of these approximations may take into account the coupling between the electronic and vibrational energy levels (vibronic coupling) that can be taken into account via the Franck-Condon or Herzberg-Teller factors. ${ }^{[140,141]}$ After light absorption, the excited state is generally in a nonequilibrium regime and thus the excess of energy induces molecular distortions that drive the system toward regions where the excited state has less potential energy, i.e. toward valleys and minima of the excited state PESs, while the excess of energy is released by means of vibrations and heat transfer to the environment. Whereas the nuclear displacements predicted for singlet and triplet MLCT states are relatively small, the vibrational relaxation of $\mathrm{MC}$ states is often ascribed to much larger distortions that move the system far from the Franck-Condon area. Important information regarding the relative energies of the MLCT and MC states and the coordinates that stabilize them can be obtained by running excited-state optimizations. As a matter of fact, the adiabatic ${ }^{3} \mathrm{MLCT} /{ }^{3} \mathrm{MC} /{ }^{5} \mathrm{MC}$ energy difference, i.e. the energy difference between the corresponding equilibrium geometries of each state defining the so-called Jablonski diagram, serves as an indicator of the MC manifold population probability. Consequently, this strategy has been used to study the photophysics of series of $\mathrm{Fe}$ (II) complexes. ${ }^{[51-53,142]}$ Figure 2 compares the adiabatic energies the ground, ${ }^{3} \mathrm{MLCT},{ }^{5} \mathrm{MLCT},{ }^{3} \mathrm{MC}$, and ${ }^{5} \mathrm{MC}$ states in the series of tridentate complexes 1-7 with different Fe-C and Fe-N coordination bonds reported by Dixon et al. ${ }^{[51]}$ The reader is referred to the original article to visualize the chemical structures. As is apparent, compound $\mathbf{6}$ exhibit degenerated energy levels between the ${ }^{3} \mathrm{MLCT}$ and ${ }^{3} \mathrm{MC}$ states. The ${ }^{3} \mathrm{MLCT} \rightarrow{ }^{3} \mathrm{MC}$ pathway will be discussed in detail in section 2.4.2.

Figure 2. Adiabatic energies of the ground ${ }^{1} \mathrm{GS},{ }^{3} \mathrm{MLCT},{ }^{5} \mathrm{MLCT},{ }^{3} \mathrm{MC}$, and ${ }^{5} \mathrm{MC}$

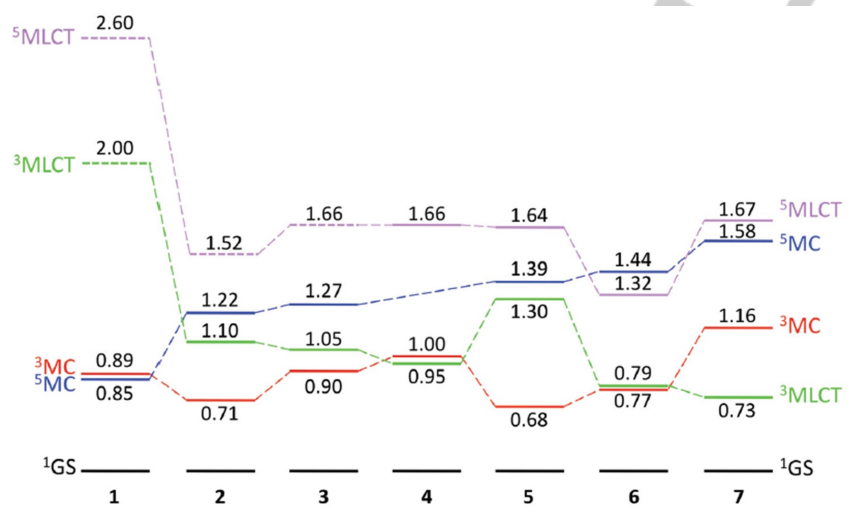

states for the 1-7 Fe(II) complexes. The chemical structures can be found in reference ${ }^{[51]}$. Reproduced from I. Dixon et al, Dalton Trans. 2015, 44, 1349813503 with permission from the Royal Society of Chemistry.

As compared to the ground-state optimization, excited states have a supplementary difficulty related to the necessity to track the correct diabatic excited state that should be optimized, i.e. MC or MLCT, while quantum chemistry codes usually provide adiabatic states and wavefunctions. This problem may lead to important instabilities in the optimization protocols leading for instance to root flipping. Sanz García et al have recently documented a new optimization algorithm which uses the natural transition orbital (NTO) formalism to track the excited-state energy during the optimization process, often a difficult task in TMCs. ${ }^{[143]}$ Even though necessary as a first step in the study of PESs, excited-state optimizations (and the obtained adiabatic energy differences) per se cannot explain in detail the intricate non-radiative photoresponse like the one occurring in $\mathrm{Fe}(\mathrm{II})$ complexes. This is due to the fact that adiabatic energy levels do not provide information concerning the crossing points between the involved PESs and the possible energy barriers that give access to these crossings. Therefore, excited-state optimizations need to be complemented by the determination of the corresponding PESs in order to characterize the different nonadiabatic events that drive the decay phenomena.

\subsection{Projected potential energy surfaces}

A first estimate to the full PESs, usually valid for relatively small geometrical deformations, can be obtained by fitting the energies obtained via excited-state optimizations and the most relevant vibrational frequencies with harmonic curves, as demonstrated by several works reported in the literature. ${ }^{[144-146]}$ The most important advantage of this strategy is that no additional quantum-chemistry calculations are required to compute the PESs, which is especially relevant in those complexes bearing large ligands and hence inducing a remarkable computational overload. However, the obtained crossing points and energy barriers remain as, sometimes rough, estimations since in general PESs do not necessarily have harmonic shape, especially in regions far from the Franck-Condon geometry like those explored by dissociative MC states.

In 2018, Lomoth, Persson, Wärnmark, and coworkers ${ }^{[55]}$ have used the projection technique to approximate the PESs in a homoleptic $\mathrm{Fe}(\mathrm{II})$ complex bearing bidentate $\mathrm{NHC}$ ligands having a record ${ }^{3}$ MLCT lifetime of 528 picoseconds (see Figure 3a). The authors ascribed the long ${ }^{3} \mathrm{MLCT}$ trapping to the presence of an energy barrier of $0.12 \mathrm{eV}$ to access the conical intersection that transfers the population to the ${ }^{3} \mathrm{MC}$ state (see Figure $3 \mathrm{~b}$ ). Therefore, even though the ${ }^{3} \mathrm{MC}$ state lies adiabatically at lower energies with respect to the ${ }^{3} \mathrm{MLCT}$ one, and one could thus expect a fast deactivation according to this energetic landscape, the estimation of the PESs and crossing points provides additional features that support the long ${ }^{3} \mathrm{MLCT}$ lifetime observed experimentally. 
a)

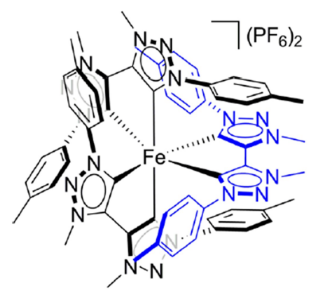

b)

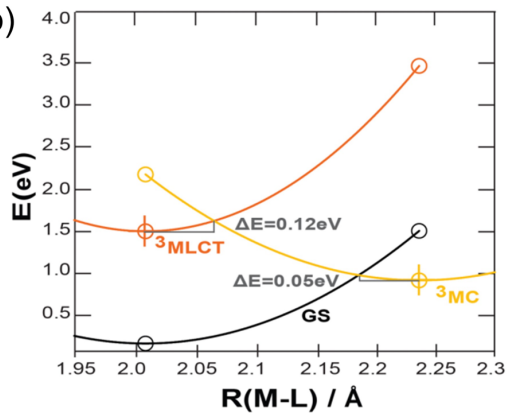

Figure 3. a) Structure of the $\left[\mathrm{Fe}(\mathrm{btz})_{3}\right]^{2+}$ complex. b) Projected PESs of the singlet ground state and lowest-lying ${ }^{3} \mathrm{MLCT}$ and ${ }^{3} \mathrm{MC}$ states of $\left[\mathrm{Fe}(\mathrm{btz})_{3}\right]^{2+}$. Adapted with permission from P. Chábera et al, J. Phys. Chem. Lett. 2018, 9 , 459-463. Copyright 2018 American Chemical Society.

\subsection{Connected paths: coordinate interpolations and relaxed scan profiles}

PESs can be explicitly computed by linearly interpolating one or several coordinates relevant for the photoprocess. Fe-ligand distances and ligand bite angles are usually the most relevant parameters that drive the excited-state decay in Fe(II) complexes, since the antibonding $e_{g}$ orbitals are populated in the ${ }^{3} \mathrm{MC}$ and ${ }^{5} \mathrm{MC}$ states, thus leading to significant bond elongation. The representation of the energy (computed by means of single-point calculations on top of the interpolated structures) as a function of the nuclear coordinates can be accurate enough to study the energy profiles of the states of interest, estimate crossing points and elucidate the operative decay mechanisms and their specific competition. ${ }^{147-149]}$ However, since the structures used in interpolated paths are not optimized, the energy barriers and the relevant crossing points must be taken as upper bounds of the actual values. The interpolated paths provide reasonably good estimates of the most important photophysical features of complex systems such as TMCs. Some works reported in the last years have combined several coordinates to construct 2D PESs, allowing a detailed understanding of the archetypal $\left[\mathrm{Fe}(\mathrm{tpy})_{2}\right]^{2+}$ [119] complex and an important Fe-NHC prototype named $\left[\mathrm{Fe}(\mathrm{CNC})_{2}\right]^{2+},{ }^{[69]}$ as displayed in Figure 4.

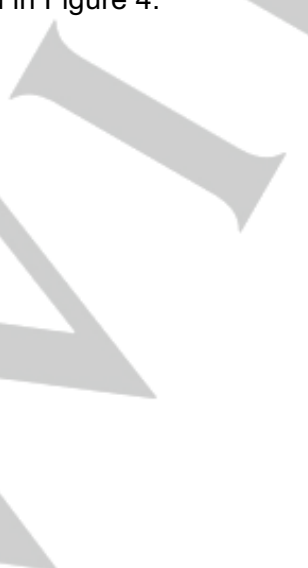

a)

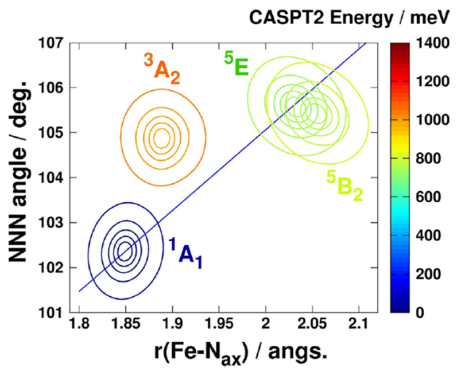

b)
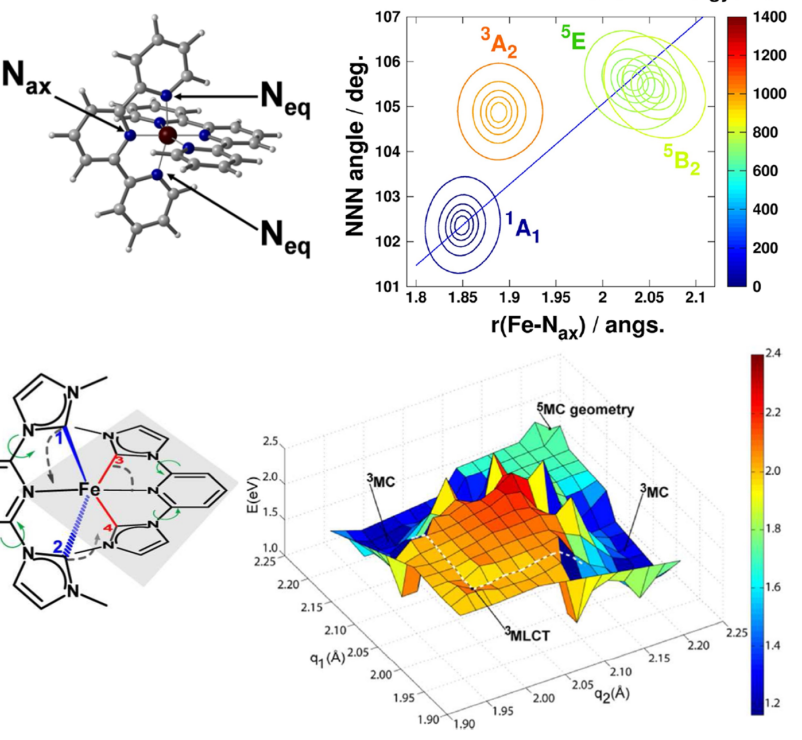

Figure 4. a) On the left side, structure of the $\left[\mathrm{Fe}\left(\mathrm{tpy}_{2}\right)_{3}\right]^{2+}$ complex and labelling of the Fe-N bonds. On the right side, CASPT2 2D PESs as a function of the NNN angle and the Fe- $\mathrm{Nax}_{a x}$ distances. Adapted with permission from M. Pápai et al, J. Chem. Theor. Comput. 2013, 9, 509-519. b) On the left side, structure of the $\left[\mathrm{Fe}(\mathrm{CNC})_{2}\right]^{2+}$ complex and definition of the $q_{1}$ (average of blue bonds) and $q_{2}$ (average of the red bonds) coordinates. On the right side, B3LYP* 2D PESs as a function of the $q_{1}$ and $q_{2}$ coordinates. The white dotted lines indicate the lowest-energy pathway between the ${ }^{3} \mathrm{MLCT}$ and the ${ }^{3} \mathrm{MC}$ states. Adapted with permission from L. A. Fredin et al, J. Phys. Chem. Lett. 2014, 5, 2066-2071. Copyright 2014 American Chemical Society.

An alternative and also relevant strategy is to linearly interpolate the path between two optimized geometries of interest, e.g. the high-spin and low-spin equilibrium structures. ${ }^{[69,119]}$ This strategy has been widely employed to study spin-crossover processes in a large variety of $\mathrm{Fe}(\mathrm{II})$ complexes. ${ }^{[148-152]}$ The PESs obtained in this way account for all the internal coordinates of the system, instead of only some of them as in the procedure described above, delivering thus more accurate paths that include larger extents of molecular relaxation. The molecular relaxation can be maximized by performing constrained optimizations on top of each interpolated structure (relaxed scan PESs) as reported by Pápai et al, ${ }^{[119]}$ although the computational cost of the protocol increases and some possible hysteresis problems may occur. Nevertheless, the latter issues can be minimized by decreasing the maximum nuclear displacement allowed at each optimization step.

PESs can also be constructed by exploring the molecular motions of the normal modes, usually computed using the harmonic approximation. ${ }^{[153]}$ The surfaces obtained in this way track the ground and excited-state energies along realistic nuclear displacements, allowing to assess the impact of specific nuclear motions on the states of interest, with the computational advantage that no additional optimizations have to be performed. Generally speaking, the main drawback of this strategy consists in the fact that the molecular vibrations at the Franck-Condon region does not necessarily represent the ones that drive the 
excited state decay, while anharmonic effects are generally neglected, and therefore the resulting paths may not represent the lowest-energy ones, i.e. the most probable mechanisms.

Jakubikova, Kelley and coworkers ${ }^{[74]}$ have employed the Smolyak's sparse grid interpolation algorithm to approximate the PESs of the $\left[\mathrm{Fe}\left(\mathrm{tpy}_{2}\right)_{3}\right]^{2+}$ complex (see Figures $4 a$ and $5 \mathrm{a}$ ) between the ${ }^{1} \mathrm{~A}$ ground state equilibrium geometry and the optimized structures of the ${ }^{1,3} \mathrm{MLCT}$ and ${ }^{3,5} \mathrm{MC}$ states. With this method, ${ }^{[154]}$ both geometries and energies can be interpolated, greatly reducing the computational cost of the study, since electronic structure calculations are only needed in a reduced number of regions. Since the complexity of the calculations grows exponentially with the number of dimensions, the authors chose three coordinates based on the main features observed in the electronic structure minimizations, namely the axial $\left(\boldsymbol{R}_{\mathrm{ax}}\right)$ and equatorial $\left(\boldsymbol{R}_{\text {eq }}\right)$ Fe-N distances and the rocking $(\Theta)$ of one tpy ligand (see Figure 5a). The method also allowed for the optimization of minimum energy crossing points (MECPs) between PESs of different spin multiplicity, as shall be discussed in section 2.4.3 in more details. As shown in Figures $5 b$ and $5 c$, the authors identified a crucial influence of the tpy rocking motion $(\Theta)$ on the adiabatic PESs.

a)

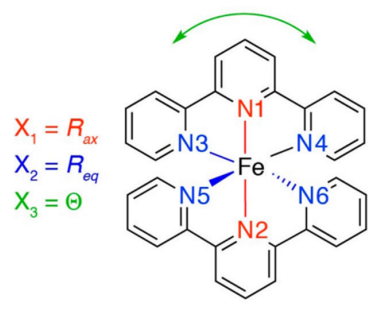

b)

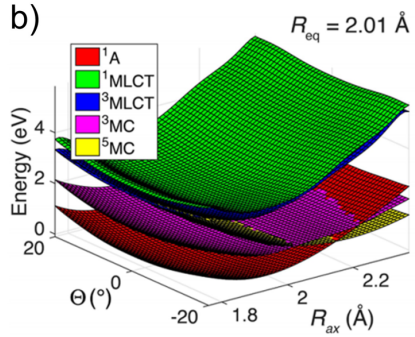

c)
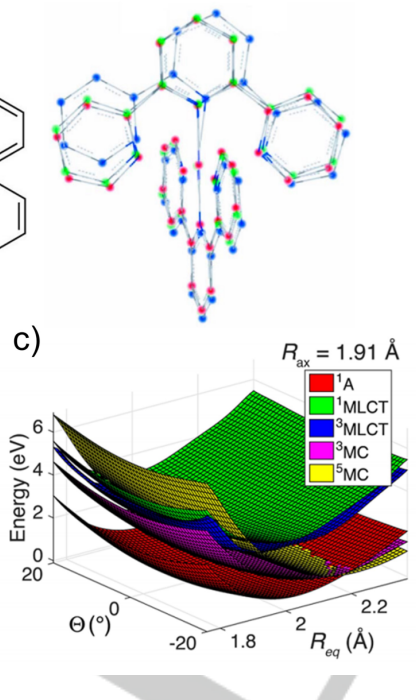

Figure 5. a) On the left side, structure of the $\left[\mathrm{Fe}\left(\mathrm{tpy}_{2}\right)_{3}\right]^{2+}$ complex and coordinates selected to construct the PESs. On the right side, optimized structures of the ${ }^{1} \mathrm{~A}$ ground state (red), ${ }^{3} \mathrm{MC}$ (green) and ${ }^{5} \mathrm{MC}$ (blue). b) and c) display the PESs obtained with the Smolyak's sparse grid interpolation algorithm fixing the $\boldsymbol{R}_{\text {eq }}$ (b) and $\boldsymbol{R}_{\mathrm{ax}}$ (c) parameters. Adapted with permission from J. Nance et al, Inorg. Chem. 2015, 54, 11259-11268. Copyright 2015 American Chemical Society.

\subsection{Minimum energy paths}

Coordinate interpolations ensure the connectivity between the points that compose the PESs, nevertheless, each point is not necessarily an energy minimum and thus linear interpolations do not represent the lowest-energy paths between the considered

PES regions. In this section, we will discuss different computational approaches that allow the determination of minimum energy paths (MEPs), in which any point is a minimum in all directions perpendicular to the path. ${ }^{[155]}$ Even if, and especially in the case of ultrafast processes and high excess of kinetic energy, other areas of higher energy can be visited, and only dynamic methods would allow to estimate their relevance, MEPs represent the most probable evolution of a chemical system. Indeed, the calculations of MEPs connecting FranckCondon regions with excited-state minima and conical intersections in the framework of the Photoreactive Path Approach (PRPA) has allowed significant breakthrough in the study of photochemical and photophysical processes of organic and inorganic systems. ${ }^{[156][157][158][159][160][161][162][163][164] ~ A s ~ p o i n t e d ~}$ out by Lindh and coworkers, "PRPA is based on mapping the path for the most efficient energy relaxation in the excited state [...] Only this procedure guarantees accurate results within the static model". ${ }^{[157]}$ The relevance of the MEPs increases in situations with low excess of kinetic energy, such as in vibrationally relaxed or "cold" excited states, where the system is forced to follow the path with the smaller energetic penalty. This approach has been widely used to study photoinduced mechanisms in organic and biochemical systems ${ }^{[165-169]}$ but relatively less used to study photoresponses in $\mathrm{Fe}(\mathrm{II})$ complexes.

We will mainly focus in two algorithms: the intrinsic reaction coordinate (IRC) and the nudged elastic band (NEB) algorithms. The choice of one or another strongly depends on the system under study, being the presence of any energy barrier along the pathway one of the most important factors to consider. Again, and due to the usual large size of TMCs, the MEP optimization algorithms are often used in combination with DFT and TD-DFT methods. This section does not aim to provide an exhaustive description of the different algorithms available to compute MEPs, but present a general overview of the procedure and the interpretation of the pathways, and discuss some recent applications in the photophysics of Fe(II) complexes. For more complete descriptions of optimization algorithms and their general applications, we refer the interested reader to thorough reviews published elsewhere. ${ }^{[170,171]}$

\subsubsection{Transition states and intrinsic reaction coordinate} algorithm

When considering only one state of a given spin multiplicity, the intrinsic reaction coordinate (IRC) represents the MEP between the TS and the corresponding two local minima (reactants or products). The determination of TSs and the associated IRCs have been widely applied to resolve reaction mechanisms occurring in the ground state. ${ }^{[172]}$ It is currently implemented in widely used quantum chemistry codes such as GAUSSIAN, ${ }^{[173]}$ MOLCAS, ${ }^{[174]}$ ORCA, ${ }^{[175]}$ TURBOMOLE, ${ }^{[176]}$ ADF, ${ }^{[177]}$ QCHEM, ${ }^{[178]}$ and GAMESS, ${ }^{[179]}$ normally compatible with both restricted and unrestricted DFT schemes (TDDFT implementations are, in general, less available). By definition, IRC determinations start from a TS, where the second derivatives for all set of coordinates $\left\{\boldsymbol{q}_{i}\right\}$ are positive except one $\left(\boldsymbol{q}_{j}\right)$ which has a negative value (eq 5): 


$$
\frac{\partial^{2} E}{\partial \boldsymbol{q}_{i}^{2}}>0 ; \frac{\partial^{2} E}{\partial \boldsymbol{q}_{j}^{2}}<0 \quad i \neq j
$$

The IRC is computed solving the differential equation (eq 6): ${ }^{[172]}$

$$
\frac{d q(s)}{d s}=v(s)
$$

where $\boldsymbol{q}$ stands for the mass-weighted Cartesian coordinates and $\boldsymbol{v}(\boldsymbol{s})$ refer to the normalized tangent vector along the IRC coordinate $s$ (eq 7):

$$
v=\frac{ \pm g}{|g|}
$$

$\boldsymbol{v}(\boldsymbol{s})$ is equal to 0 at the TS structure $(\boldsymbol{s}=0)$, negative for $\boldsymbol{s}>0$ and positive for $s<0$, while $g$ stands for the mass-weighted gradient vector. ${ }^{[172]}$ The resolution of eq 6 at each IRC step within a defined hypersphere moves the system along $v(s)$ and ensures the connectivity between the converged points, allowing the determination of the IRC. The IRC algorithm computes the downhill evolution of the system until a barrier along the path is found. Usually, and due to the high sensitivity of the algorithm, very small energy barriers are sufficient to stop the calculation and hence terminate the IRC profile. In these cases, independent standard optimization algorithms, and eventually coordinate interpolation techniques, may be used to connect the last IRC point with the global minimum.

In principle, the conventional use of the IRC algorithm could be applied to calculate singlet, triplet or quintet PESs of $\mathrm{Fe}(\mathrm{II})$ complexes displaying energy barriers, eventually computing the open-shell wave functions via unrestricted DFT methods. A prerequisite is however to characterize the corresponding TS by converging a structure with a unique imaginary frequency, which remains a difficult task considering that often complex coordinates are involved in the PESs, hampering the convergence of standard single-ended TS optimization algorithms ${ }^{[171]}$ to correct structures. In these cases, TS areas should be explored using methods such as NEB or related procedures, as discussed in section 2.4.4.

2.4.3. Intrinsic reaction coordinate algorithm and minimum-energy crossing point optimizations

The situation is different when considering barrierless profiles or states of different spin multiplicities that may cross. If two points of interest on an excited state PES are connected via a downhill path, the IRC algorithm can be used starting from the highestenergy point to compute the downhill MEP toward the nearest local or absolute minimum. The IRC algorithm (usually called MEP algorithm when it does not start from a TS structure) solves eq 6 at each point to map the lowest-energy PES of the state of interest. The concept is illustrated in Figure 6. In a), a minimum in a state labelled as "high spin" (for instance, a ${ }^{5} \mathrm{MC}$ equilibrium geometry) is connected with a minimum of another state labelled as "low spin" state (e.g. ${ }^{3} \mathrm{MLCT}$ or $\mathrm{S}_{0}$ equilibrium geometries) by relaxing the low-spin state (MEPL). Due to the absence of energy barriers in the high- to low-spin state pathway (left to right), the energy difference between the high- and low-spin states $\left(\Delta \boldsymbol{E}_{\boldsymbol{H}}\right)$ is equal to the activation energy required to transfer population from the low-spin state to the high-spin state $\left(\Delta E_{L \rightarrow H}^{ \pm}\right)$:

$$
\Delta E_{H L}=E_{H}-E_{L}=\Delta E_{L \rightarrow H}^{\ddagger}
$$

and thus, the MEP itself represents the energy barrier $\Delta \boldsymbol{E}_{L \rightarrow H}^{\neq}$, since $\Delta \boldsymbol{E}_{H \rightarrow L}^{\ddagger}=\mathbf{0}$. This path yields an accurate mechanism for the intersystem crossing phenomenon that does not depend on the choice of any coordinate, i.e. the structural changes are obtained by following the nuclear gradient evolution. Providing optimized paths along the PES is also important to interpret transient excited-state absorption (ESA) spectra, which mostly takes place along the most probable (lowest-energy) excited-state PES.

In the specific example shown in Figure 6a, the MECP between the two PES of different spin multiplicity coincides with the minimum of the high-spin state. This is not necessarily a general case, and one can calculate a MECP relatively far away from the equilibrium geometries of the two states (see the example drawn in Figure $6 \mathrm{~b}$ ). In these cases, the approximated MECP between the two states (hereafter denoted as MECP') can be determined by computing the MEPL starting from the equilibrium geometry of the high-spin state. Since the MEP relaxing the low-spin state $\left(M E P_{L}\right)$ represents an optimized path for the low-spin state that connects both high-spin and low-spin state minima, the MECP' point obtained in this way quantifies the activation energy $\Delta \boldsymbol{E}_{L \rightarrow H}^{\neq}$ needed to induce the spin-crossover. Even though specific MECP optimizations taking into account both high-spin and low-spin gradients (see below) could eventually provide MECPs at lower energies found at different regions of the PESs, the latter points do not necessarily guarantee a connectivity between the two highspin and low-spin minima as displayed in Figure 6b. A recent application of this MEP strategy to study the influence of the facial 
$(\mathrm{fac}) /$ meridional (mer) structural isomerism in the PESs of Fe(II)$\mathrm{NHC}$ complexes will be explained in detail in section 3 .
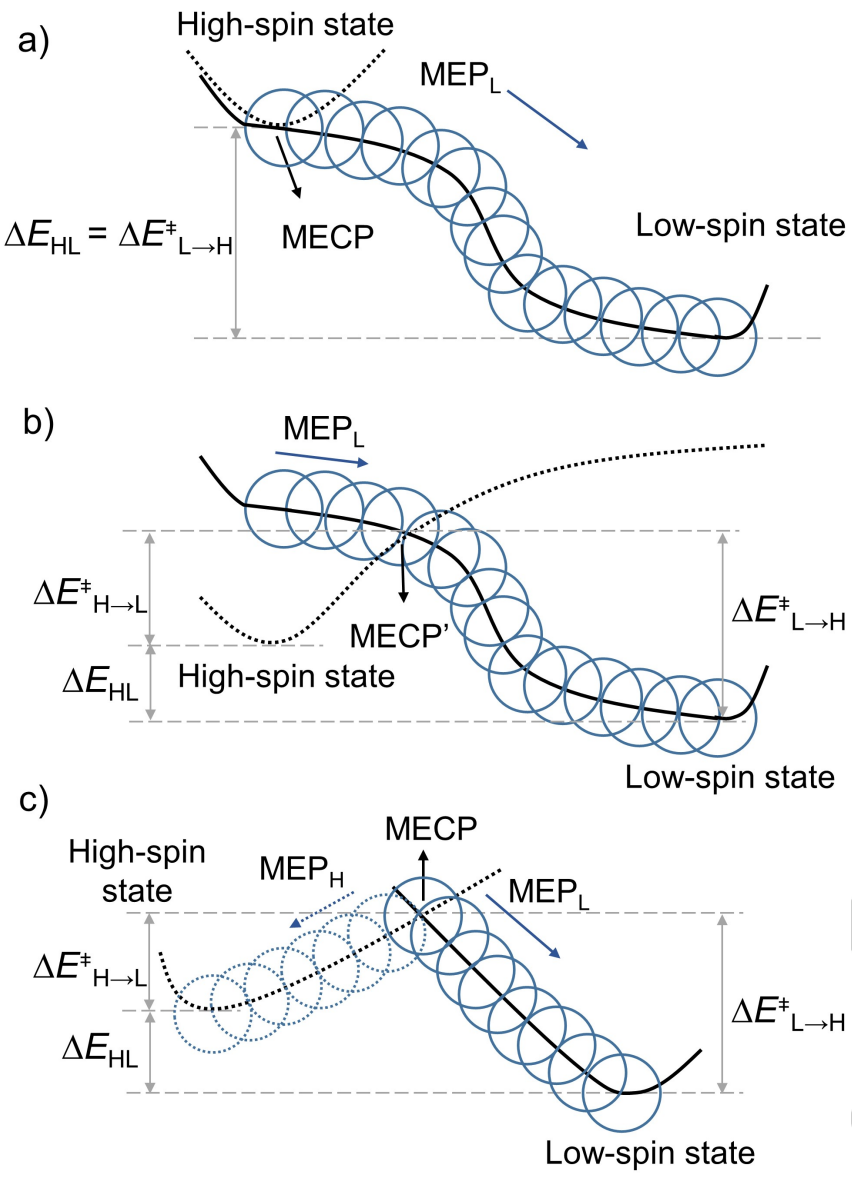

Figure 6. a) MEP profile connecting two points on the same PES in a barrierless manner. b) Determination of a relevant MECP' by means of MEP calculations. c) High-spin and low-spin MEPs that connect the two corresponding equilibrium geometries through a high-energy MECP. MEPL and MEP $P_{H}$ indicate that the lowand high-spin states is relaxed, respectively, $\Delta E_{H L}$ stands for the high-low spin adiabatic energy difference, whereas $\Delta E_{L \rightarrow H}^{ \pm}$and $\Delta E_{H \rightarrow L}^{ \pm}$refer to the activation energy for the low-spin to high-spin population transfer and vice versa, respectively, and MECP stands for minimum energy crossing point. Low-spin and high-spin PESs are represented with solid and dotted lines, respectively. The blue circles represent the MEP hyperspheres at each optimization step.

A third case consist on the use of MECP optimization algorithms to obtain the minimum-energy structure exhibiting energy degeneracy between the two states of interest (conical intersections, singlet-triplet crossings, ${ }^{[180,181]}$ as displayed in Figure 6c. These algorithms are implemented, for example, in the Graphical User Interface (GUI) of the ADF2018 modelling suite, ${ }^{[177]}$ ORCA, ${ }^{[175]} \mathrm{Q}-\mathrm{Chem},{ }^{[178]}$ and in the MECPro code ${ }^{[182]}$ (which is interfaced with GAUSSIAN 09 ${ }^{[173]}$ ). However, as stated before, these MECPs algorithms may explore regions of the PES far from the equilibrium and thus their connectivity with the equilibrium geometries of interest should be checked to ensure the photochemical relevance of the MECPs. Coordinate interpolations or, ideally, MEP calculations as displayed in Figure $6 \mathrm{c}$, can provide the fully connected PESs. Computing the MEP relaxing the high- $\left(\mathrm{MEP}_{\mathrm{H}}\right)$ or low-spin $(\mathrm{MEP})$ states departing from the converged MECP allows the determination of the optimized PESs that mediate the intersystem crossing processes. In the example shown in Figure 6c, the relative energy of the states $\Delta \boldsymbol{E}_{H L}$ does not provide a good estimation for the intersystem crossing kinetics, since one should consider the energy barriers to access the MECP (eq 9):

$$
\Delta \boldsymbol{E}_{H L} \neq \Delta \boldsymbol{E}_{L \rightarrow H}^{\ddagger} \neq \Delta \boldsymbol{E}_{H \rightarrow L}^{\ddagger}
$$

This was noted by Dixon and coworkers, who computed the $\mathrm{S}_{0} /{ }^{3} \mathrm{MC}, \mathrm{S}_{0} /{ }^{5} \mathrm{MC}$, and ${ }^{3} \mathrm{MC} /{ }^{5} \mathrm{MC}$ MECPs of the $\left[\mathrm{Fe}(\mathrm{tpy})_{2}\right]^{2+}$ and $\mathrm{Fe}(\mathrm{CNC})_{2}$ complexes (see Figure 7). ${ }^{[183]}$ The MECP optimizations revealed the presence of energy barriers for the intersystem crossing processes up to $0.38 \mathrm{eV}$, which play a crucial role in the excited-state decay kinetics and therefore must not be neglected when studying photoinduced processes in TMCs. On the other hand, Nance et al validated the use of approximated PESs by means of the Smolyak's sparse grid interpolation algorithm to estimate MECPs. ${ }^{[74]}$
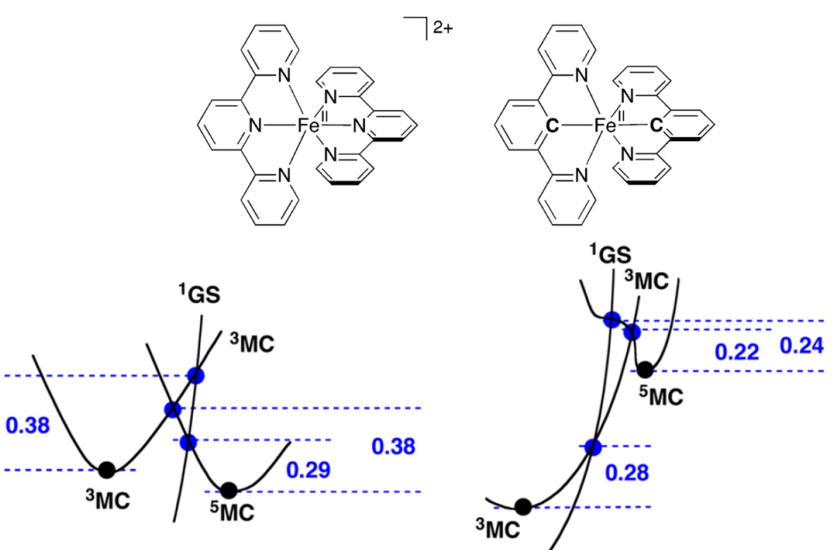

Figure 7. Chemical structures of the $\left[\mathrm{Fe}(\mathrm{tpy})_{2}\right]^{2+}$ (left) and $\mathrm{Fe}(\mathrm{CNC})_{2}$ (right) complexes and their corresponding MECPs (values in blue). Adapted with permission from I. Dixon et al, Inorg. Chem. 2013, 52, 13369-13374. Copyright 2013 American Chemical Society.

Especially for intersystem crossing processes, the determination and analysis of the energy barriers to access the MECPs (see Figures 6 and 7) should be accompanied by the study of the coupling between the involved states. In cases of large couplings, the diabatic states will be largely superposed giving rise to adiabatic states of highly mixed nature. Hence, the transitions can take place continuously through the adiabatic evolution of the mixed PES. On the contrary, in cases of low couplings, the mixing will be negligible and the transition between the states will happen abruptly in non-adiabatic frameworks, in which the nuclear wavepacket will split between the two PESs with a branching ratio depending on their coupling, energy difference and PES topologies.

Whereas for internal conversions between states of the same spin multiplicity the non-adiabatic coupling between the involved PESs is generally assumed to be relatively large when the energy 
difference between the PESs is sufficiently small, giving rise to probable non-adiabatic jumps, the situation is different when considering population transfer between states of different spin multiplicities. The inter-system crossing probability is directly related to the inverse of the energy difference and to the spin-orbit coupling (SOC) between the states of interest, and thus this SOC has to be considered in the analysis. A detailed description of the several methods to determine SOCs, usually necessitating a perturbative treatment of the relativistic effects, is out of the scope of the present review. For further details, the interested reader is referred to a recent survey published elsewhere. ${ }^{[184]} A$ few quantum-chemistry codes (ADF 2018,,$^{[177]}$ ORCA 4.0, ${ }^{[175]}$ DALTON16 $\left.{ }^{[185]}\right)$ have implemented singlet-triplet SOC calculations at the TD-DFT level, whereas with ab initio multiconfigurational methods the implementations are in general more available. As a general principle, the inclusion of relativistic effects and SOCs may have important effects on the energy of the different states, and hence on the MEP and barrier heights. However, in the case of the relatively light iron, SOCs are in the order of $\sim 100 \mathrm{~cm}^{-1}(\sim 0.01 \mathrm{eV})$ and therefore those effects should be considered as negligible.

In analogy with transition state theory (TST), widely used to study ground-state chemical reactivity, some approaches have been developed to calculate kinetic rates of non-adiabatic or spinforbidden reactions from static quantities. The generalization of TST usually involves taking into account the energy barrier necessary to access singlet-triplet crossings or conical intersections together with the effective number of rovibrational states allowing the crossing. The latter quantity is estimated taking into account the energy distribution of the rovibrational levels embedded in the two electronic states and the crossing probability via the non-adiabatic couplings and the SOCs. Despite the fact that the former approach may represent a rather crude approximation, it has been shown to provide accurate enough estimations of the reaction rates, with errors restricted to an order of magnitude, at a much reduced computational cost as compared to dynamic approaches. As such, the strategy has been applied to a number of photophysical and photochemical processes involving TMCs and including iron-sulfur proteins such as [NiFe]hydrogenase, ferredoxin, and rubredoxin. ${ }^{[186,187]}$

\subsubsection{Nudged elastic band method}

As previously said, optimizing TSs in the excited state using the Quasi-Newton and related methods can be problematic since, in $\mathrm{Fe}$ (II) complexes, as in many other systems, the reaction coordinate is often complex and involves coupled variations in bond lengths, angles and dihedrals. Usual single-ended TS optimization algorithms only explore a relatively small region of the PESs, which strongly depends on the initial guess provided by the user. The NEB method aims to solve this problem. The whole pathway is initially sampled by generating a set of equidistant structures or images through coordinate interpolations between the reactant and the product (see Figure 8). ${ }^{[155]}$ These images, which can be interpreted as a guess of the MEP, define an elastic "band" since a spring potential is added between the points. ${ }^{[171]}$ Each image is subsequently optimized by following the NEB force $\boldsymbol{F}_{\boldsymbol{i}}^{\boldsymbol{N} E \boldsymbol{B}}$ (eq 10):

$$
F_{i}^{N E B}=F_{i}^{\perp}+F_{i}^{S \|} \quad(10)
$$

where $F_{i}^{\perp}$ is the component due to the curvature of the PES perpendicular to the band, and $\boldsymbol{F}_{i} \|$ refers to the force parallel to the band (see Figure 8). ${ }^{[155]}$ The convergence of the multiple optimizations provides the MEP with a resolution that depends on the number of images used to construct the elastic band. Since the initial and the final structures are required to start the procedure, methods like the NEB are called double-ended methods. ${ }^{[188]}$ They represent interesting approaches to compute the MEP of complex photophysical paths that may involve several TS structures.

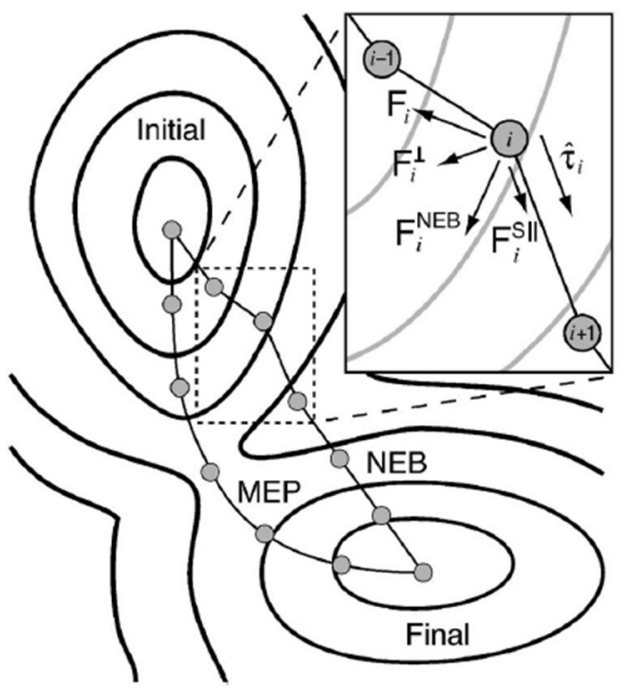

Figure 8. Schematic representation of the NEB method. Reprinted from D. Sheppard et al, J. Chem. Phys. 2008, 128, 134106 with the permission of AIP Publishing.

Regarding the availability of the NEB method and related approaches, it is implemented in a variety of quantum-chemistry codes such as TURBOMOLE, ${ }^{[176]}$ ORCA4.0, ${ }^{[175]} \mathrm{Q}-\mathrm{CHEM}^{[178]}$ and TERACHEM. ${ }^{[189]}$ The latter program runs in GPUs instead of standard CPUs, dramatically reducing the computational cost of the jobs.

Dixon et al used the NEB method to determine the energy barrier for the ${ }^{3} \mathrm{MLCT} \rightarrow{ }^{3} \mathrm{MC}$ path for a $\mathrm{Fe}(\mathrm{II})$ complex for which the adiabatic energies of both triplet states are degenerated. ${ }^{[51]}$ The energy levels are displayed in Figure 2 and correspond to the complex 6 . The MEP for the aforementioned process revealed a significant energy barrier of $\sim 0.2 \mathrm{eV}$ (see Figure 9), which could trap the system in the initially populated ${ }^{3} \mathrm{MLCT}$ state. Indeed, the authors suggested a ${ }^{3} \mathrm{MLCT} \rightleftharpoons{ }^{3} \mathrm{MC}$ equilibrium process highly influenced by the deactivation of the ${ }^{3} \mathrm{MC}$ state through its corresponding crossing with the ground state. To assess the competition between the processes, the ${ }^{3} \mathrm{MC} / \mathrm{S}_{0} \mathrm{MECP}$ was 
optimized and found at $0.18 \mathrm{eV}$ above the ${ }^{3} \mathrm{MC}$ minimum, pointing to a competitive channel also depending on the SOC magnitude between the ${ }^{3} \mathrm{MC}$ and the $\mathrm{S}_{0}$ states. ${ }^{\left[{ }^{15]}\right.}$ Once again, the determination of PESs and MECPs was shown to be crucial in providing accurate reaction paths able to describe the main photophysical features of the photoinduced processes.

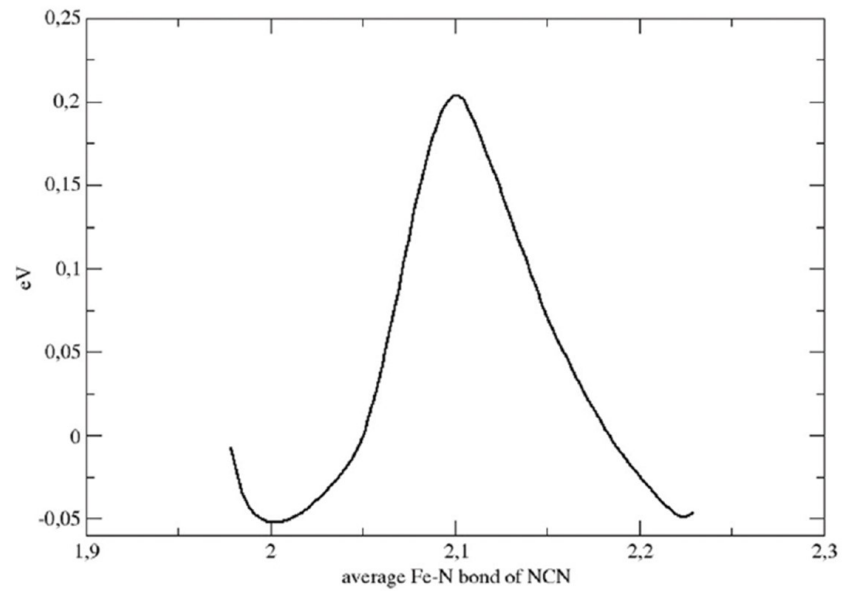

Figure 9. MEP of a $\mathrm{Fe}(\mathrm{II})$ complex with a degenerated ${ }^{3} \mathrm{MLCT}$ and ${ }^{3} \mathrm{MC}$ adiabatic energies obtained with the NEB method. Reproduced from I. Dixon et al, Dalton Trans. 2015, 44, 13498-13503 with permission from the Royal Society of Chemistry.

\section{Case study: impact of the fac/mer isomerism in the potential energy surfaces of bidentate $\mathrm{Fe}$ (II)-NHC complexes}

As illustrated in the previous sections, many different efforts have been devoted to clearly elucidate the interplay between iron complexes' PES and their possible exploitation as optically active materials. As a matter of fact, most of the computational and experimental studies have addressed tridentate $\mathrm{Fe}$ (II) complexes and the crucial role of NHC ligands in increasing the ${ }^{3} \mathrm{MLCT}$ lifetimes has been unambiguously evidenced. The present section discusses a recent case study concerning the elucidation of the PESs of two isomers of a bidentate pyridil-carbene $\mathrm{Fe}(\mathrm{II})$ complex reported by our research group (see Figure 10), ${ }^{[57]}$ leading to unexpected results concerning fundamental photophysical mechanisms. The less pronounced geometrical deformation from an ideal octahedral arrangement as compared to tridentate compounds, was believed to bring important effects in allowing an optimal energy level alignment and hence in increasing the overall ${ }^{3} \mathrm{MLCT}$ lifetime with respect to the $[\mathrm{Fe}(\mathrm{bpy}) 3]^{2+}$ prototype (see Figure 1).

However, and differently from the case of tridentate compounds, asymmetric bidentate ligands lead to the coexistence of two isomers, namely the fac and mer arrangements. In the case of $\mathbf{C 1}$ complex (see Figure 10), due to statistical preferences during the synthetic procedure, the two isomers are not equivalently produced and the complex is obtained as a mixture with a 1:14 fac/mer ratio (see structures in Figure 10). a)

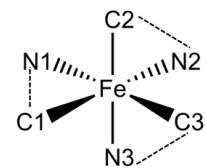

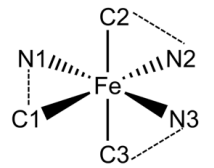

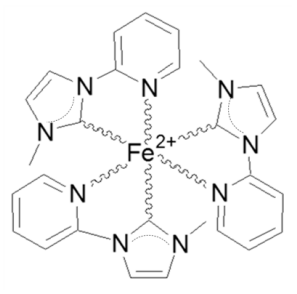

C1

b)

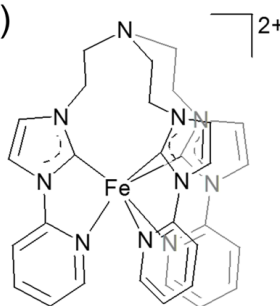

C2
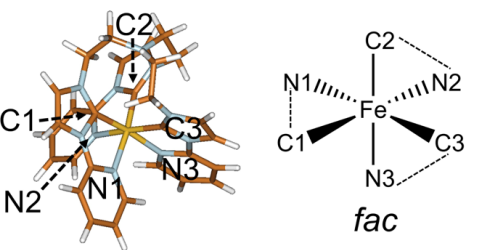

fac
Figure 10. Structure of $\mathbf{C 1}$ (a) and $\mathbf{C 2}$ (b) complexes and the fac and mer isomers. Adapted from A. Francés-Monerris et al, Inorg. Chem. 2018, 57, 10431-10441. Copyright 2018 American Chemical Society. Adapted from K Magra et al, Inorg. Chem. 2019, 58, 5069-5081. Copyright 2019 American Chemical Society.

\subsection{Absorption properties of the fac and mer isomers of $\mathrm{C}_{1}$}

The optical and photophysical properties of both $\mathbf{C} \mathbf{1}$ isomers were rationalized owing to DFT and TD-DFT based molecular modeling of the different excited state, involving both the singlet and triplet state manifolds. While the ground state geometries were obtained at the B3LYP level, all excited states have been described by using the HCTH functional. This choice was dictated by the fact that this functional provides a good description of the excited state manifold of similar iron complexes. ${ }^{[50,52,57,64]}$ Indeed, and despite a systematic shift of $0.28 \mathrm{eV}$ toward the blue was applied to the excitation energies to facilitate the comparison with the experimental recordings, the order and the nature of the states is correctly provided by this functional, as highlighted by using the NTO formalism. ${ }^{[190,191]}$ The absorption spectrum of both isomers and of the mixture was simulated taking into account the effects of thermal and vibrational motion by a Wigner distribution sampling of the ground state equilibrium region. The absorption in the visible region is, as expected, dominated by the presence of a large number of ${ }^{1} \mathrm{MLCT}$ states, while ${ }^{1} \mathrm{MC}$ states are computed at higher energies. More importantly, by comparing the fac and mer spectra, and despite the global similarity between the two isomers, one can note the emergence of some subtle differences, in particularly concerning the MLCT density of states.

\subsection{Decay to the lowest-lying excited states}


When studying the intricate photoresponse of TMCs in general and $\mathrm{Fe}(\mathrm{II})$ complexes in particular, an important step is the elucidation of the role played by the lowest-lying excited states since often only the higher-energy states are populated upon light absorption. Even though in the literature it is usually assumed that the lowest-lying excited states dominate the decay (Kasha's rule), ${ }^{[51,55,192-194]}$ the study of the "vertical" relaxation by identifying the coordinates that can have a strong influence in the highenergy electronic states is a crucial step to rule out possible events occurring in the high-energy manifold, and thus safely rely on the description of the lowest-lying PESs to explain the photoinduced responses taking place in the complexes.

For both fac/mer isomers of $\mathbf{C 1}$, a high density of singlet and triplet states below the bright ${ }^{1} \mathrm{MLCT}$, that can assumed to be populated upon visible irradiation, is present both at Franck-Condon and at the $S_{1}$ minimum, hence questioning the first steps of the relaxation pathways. To ascertain the vertical relaxation toward the lowestlying states, the magnitude of the SOCs were determined at the So minima and the energy of the high-lying states was tracked upon the Fe-N bond stretch provided by MEP calculations relaxing the $T_{1}$ state. On the one hand, the SOC elements are quite high, as expected for MLCT states, reaching $130 \mathrm{~cm}^{-1}$. On the other hand, analyses of the PESs of the triplet states along the MEP relaxing the $T_{1}$ state revealed that only the first three triplet states are stabilized upon the MEP coordinates, while the high-energy states are clearly dissociative (see Figure 11). In addition, the PESs for the $T_{1}$ and $T_{2}$ states are almost parallel.

Taking into account the high density of states, the SOC values and the geometrical changes it was concluded that the intersystem crossing takes place during the first vibrational cooling following the excitation. This is also in agreement with results from UV/Vis and X-ray time-resolved absorption spectroscopy studies establishing the intersystem crossing to take place in around 100 fs for related iron compounds. ${ }^{[50,52,63,64]}$ All in such a scenario, and especially the observed high density of states, corroborates the use of the Kasha's rule, stipulating that the whole photophysics and photochemistry can be rationalized by exploring only the PESs of the lowest-energy excited states of c1.

In principle, other photochemical pathways involving different MC states may be possible as highlighted for $\operatorname{Ru}(\mathrm{II})$ and $\operatorname{Ir}(\mathrm{III})$ complexes, ${ }^{[195-197]}$ that may in addition be strongly dependent on the isomerism. However, the fact that all the high-lying triplet states are dissociative (see Figure 11) points to the fact that, at least for this family of iron complexes, the photohysical relaxation involving deactivation toward the low-lying ${ }^{3} \mathrm{MC}$ states is dominant.

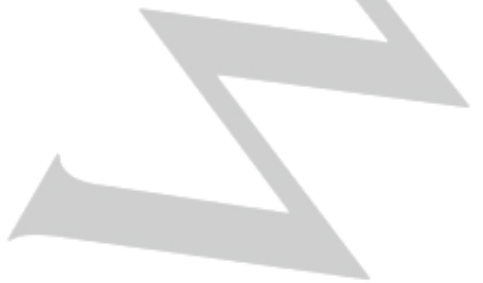

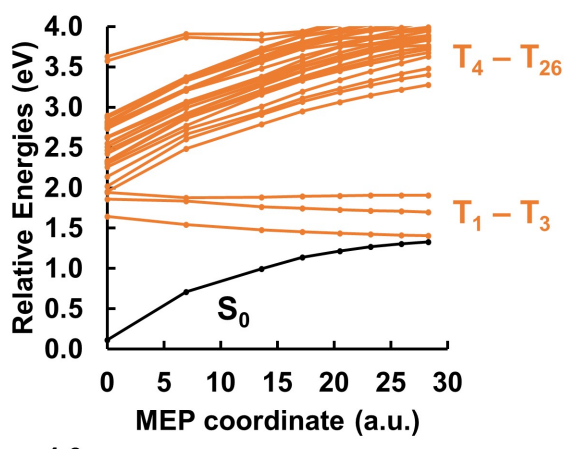

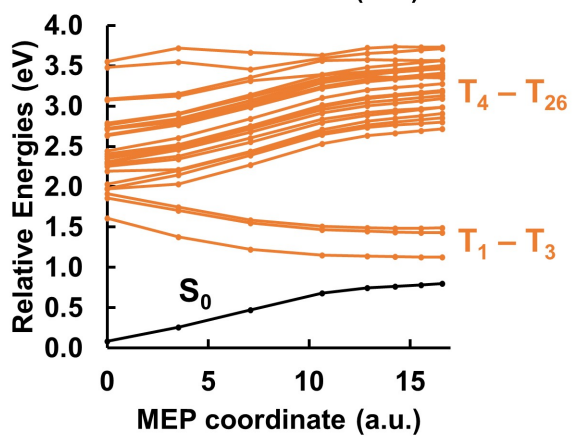

Figure 11. Energies of the first 26 triplet states of the fac (top) and mer (bottom) isomers of $\mathbf{C} 1$ along the MEP coordinate relaxing the $T_{1}$ state. Note that the absorption takes place in a window comprised between 2.5-3.0 eV. Adapted from A. Francés-Monerris et al, Inorg. Chem. 2018, 57, 10431-10441. Copyright 2018 American Chemical Society.

\subsection{Potential energy surfaces and influence of the isomerism}

Due to highly prominent presence of the mer isomer in the synthetic mixture of $\mathbf{C 1}$, the study of the specific impact of the isomerism in the PESs of the bidentate complex C1 (Figure 10a) required the synthesis of $\mathbf{C 2}$, characterized by a tripodal constrained arrangement (Figure 10b) that prevents the formation of mer isomers. ${ }^{[57,198]}$ Note that the photophysical properties and the PESs of $\mathbf{C} 2$ and the fac isomer of $\mathbf{C} 1$ are equivalent, as proven by the very similar singlet, triplet, and quintet energies, the relatively planar triplet PESs and the globally similar photochemical landscape. ${ }^{[57,198]}$

The singlet, triplet and quintet PESs leading to the Fe-N dissociations were built by computing the $T_{1}$ MEP using the IRC algorithm, as described in section 2.4.2, from the ${ }^{3} \mathrm{MLCT}$ to ${ }^{3} \mathrm{MC}$ minima (left part of the curves). The full PES landscape for the mer-C1 isomer is displayed in Figure 12, whereas the PESs for C2, which represents a pure fac arrangement, is displayed in Figure 13. First, it is important to underline that the conversion from MLCT to MC nature proceeds adiabatically through a highly mixed region instead than via a non-adiabatic transition mediated by singularities and crossing points. This is a similar case to the general example displayed in Figure 6a, in which the state represented with the solid lines correspond to $T_{1}$, the state represented with dashed lines correspond to $S_{1}$, and where both 
states do not cross at the left side of the curve since $S_{1}$ lies at higher energies (see Figures 12 and 13). Thereby, the MEP profile represents the lowest-energy adiabatic ${ }^{3} \mathrm{MLCT} \rightarrow{ }^{3} \mathrm{MC}$ transition for $\mathbf{C 1}$ and $\mathbf{C 2}$, mainly driven by the stretch of the Fe$\mathrm{N} 1$ bond (see Figure 10 for atom labelling).

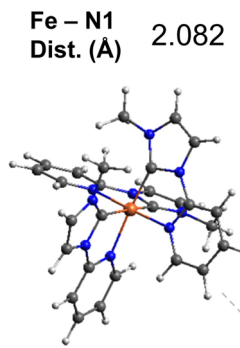

$\mathrm{S}_{0} \min$
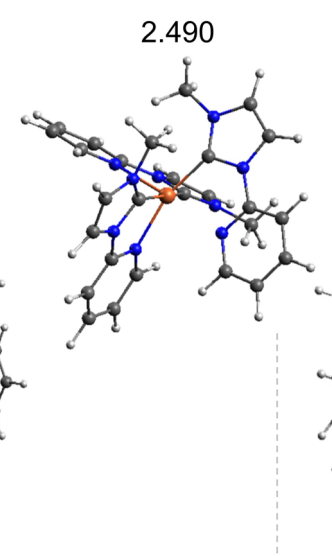

2.334

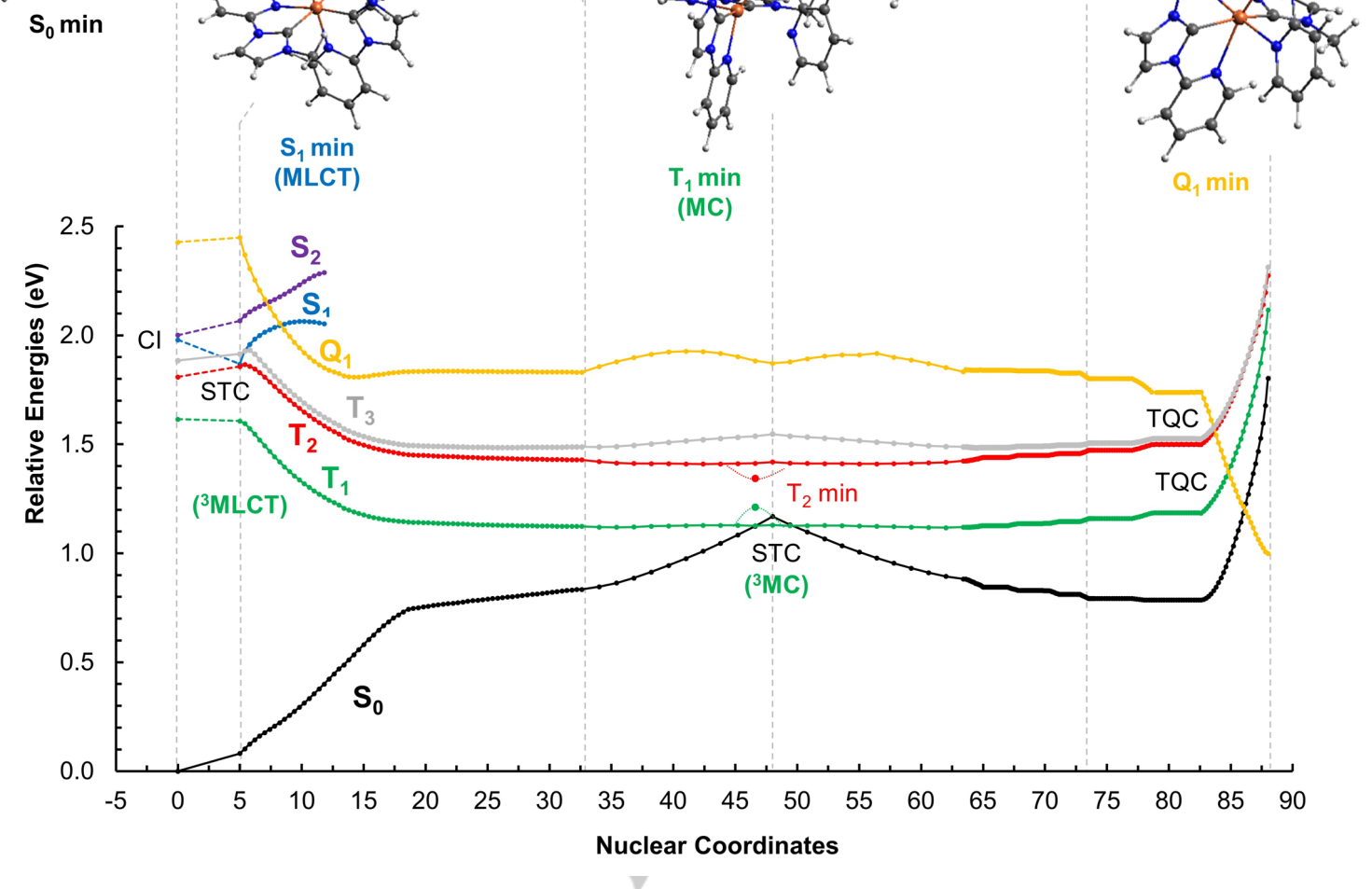

Figure 12. Lowest-lying singlet, triplet and quintet PESs for the mer isomer of $\mathbf{C} 1$. The nuclear coordinates $0,5.0,48.0$, and 88.0 correspond to the $\mathrm{S}_{0}$ min, $\mathrm{S}_{1}$ min $\left({ }^{1} \mathrm{MLCT}\right), \mathrm{T}_{1} \mathrm{~min}\left({ }^{3} \mathrm{MC}\right)$, and $\mathrm{Q}_{1} \mathrm{~min}$, respectively. $\mathrm{T}_{1}$ and $\mathrm{T}_{2}$ energies at the $\mathrm{T}_{2} \mathrm{~min}\left({ }^{3} \mathrm{MC}\right)$ structure (nuclear coordinates $\left.=46.6\right)$ are also shown. $\mathrm{Cl}=\mathrm{conical}$ intersection, STC = singlet-triplet crossing, TQC = triplet-quintet crossing. Reproduced from A. Francés-Monerris et al, Inorg. Chem. 2018, 57, $10431-10441$. Copyright 2018 American Chemical Society.

Even though the MEP profiles are globally similar for the two isomers, important differences arise and should be properly interpreted. Indeed, the fac isomer presents globally flat $T_{1}$ and $T_{2}$ PESs all along the MEP coordinate, while the mer isomer has much steeper profiles close to the ${ }^{3} \mathrm{MLCT}$ minimum, pointing to a much larger vibrational relaxation for the latter as compared to the fac arrangement. This implies important differences in the singlettriplet crossing areas that in turn will induce important differences in the photophysical properties, as discussed in the next sections.

The right side part of the PESs shown in Figures 12 and 13 was computed by MEP calculations relaxing the $T_{1}$ state starting from the ${ }^{5} \mathrm{MC}\left(\mathrm{Q}_{1}\right)$ equilibrium structure toward the ${ }^{3} \mathrm{MC}$ minimum. This pathway represents an example of the general case displayed in Figure $6 \mathrm{~b}$, in which the low-spin state is $\mathrm{T}_{1}$ and the high-spin state corresponds to $Q_{1}$, and the MECP' obtained is a photochemically relevant $T_{1} / Q_{1}$ triplet-quintet crossing. For both isomers, a significant participation of the quintet states to the overall decay process was safely excluded due to the high energy values of the crossings and the associated energy barriers, avoiding its population from the ${ }^{3} \mathrm{MC}$ minima. 


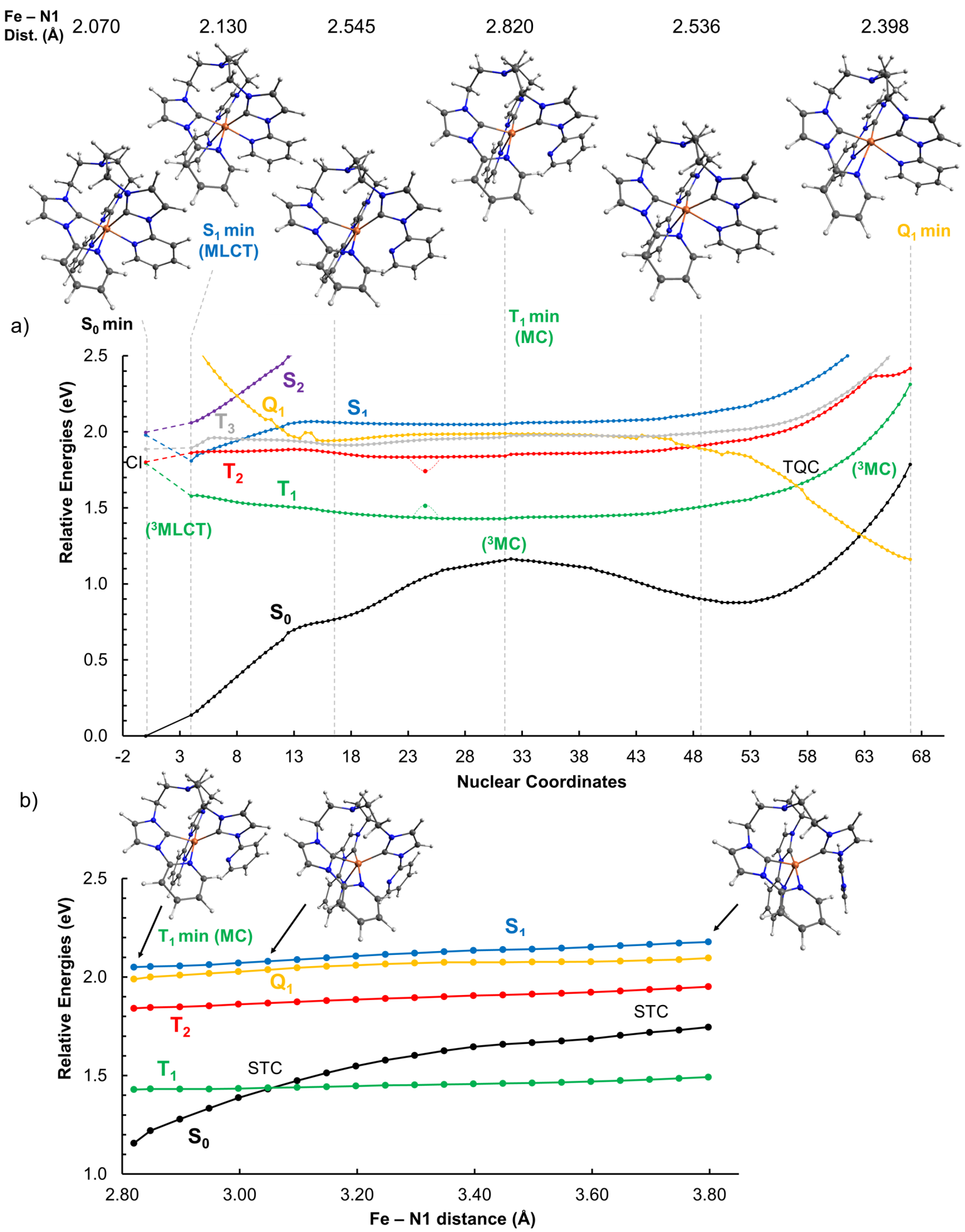

Figure 13. a) Lowest-lying singlet, triplet and quintet PESs for C2. The nuclear coordinates $0,4.0,31.5$, and 67.0 correspond to the $\mathrm{S}_{0} \mathrm{~min}, \mathrm{~S}_{1} \mathrm{~min}\left({ }^{1} \mathrm{MLCT}\right.$ ), $\mathrm{T}_{1} \mathrm{~min}$ $\left({ }^{3} \mathrm{MC}\right.$ ), and $Q_{1}$ min, respectively. $T_{1}$ and $T_{2}$ energies at the $T_{2} \min \left({ }^{3} \mathrm{MC}\right)$ structure (nuclear coordinates $=24.5$ ) are also shown. b) Energies of the most relevant states mapped upon Fe-N1 bond stretching by means of relaxed scan calculations on the $\mathrm{T}_{1}$ surface starting from the $\mathrm{T}_{1} \min \left({ }^{3} \mathrm{MC}\right)$ geometry of $\mathbf{C 2}$. Cl $=$ conical intersection, STC = singlet-triplet crossing, TQC = triplet-quintet crossing. Reproduced from K. Magra et al, Inorg. Chem. 2019, accepted. Copyright 2019 American Chemical Society. 
Finally, and for both iron-based isomers, the coordinate leading to the singlet-triplet crossing and hence to the deactivation of the triplet state via repopulation of the ground state was clearly identified as the non-symmetric increase of the Fe-N distance (Figure 13b). Unveiling the Fe-N distance as the leading vibrational mode to induce the excited state decay, at least for bidentate iron complexes, is of extreme importance since its rigidification via steric constrains or $\pi$-stacking could be a viable strategy to significantly increase the lifetimes of the chargetransfer states.

\subsection{Interpreting the excited-state lifetimes using the potential energy surfaces}

From the results reported in the previous section, it is clearly evident that $\mathrm{fac} / \mathrm{mer}$ isomerism is far from being innocent in shaping the PES topologies. The differences found in the energy profiles can be used to rationalize the photoresponses of both $\mathbf{C} 1$ and $\mathbf{C 2}$ iron complexes measured experimentally.

Time resolved absorption spectroscopy revealed that the MLCT decay of the $\mathbf{C 1}$ mixture dominated by the mer isomer is characterized by two components with different timescales: a fast component with characteristic time $\tau_{2}=2-3 \mathrm{ps}$, and a slow one with $\tau_{3}=15-20 \mathrm{ps}$. In the case of the constrained fac-like tripodal compound C2, a similar picture can be drawn, with once again two processes having two distinct timescales $\tau_{2}=3-4 \mathrm{ps}$, and $\tau_{3}=$ $15-20$ ps. Interestingly, for both compounds the two pathways are not sequential but are instead taking place in parallel, as clearly demonstrated by the analysis of the differential absorption.

The main differences between the two isomers are $i$ ) the slower $\tau_{2}$ component for the pure fac $\mathbf{C 2}$, and ii) the different branching ratio, and hence the probability, of the two decay pathways. Indeed, while for the fac arrangement the fast/slow branching ratio is of $57 \%$, for the mer isomer it accounts for $87 \%$. In both isomers, the fast component was ascribed to the decay of the $T_{2}$ state (through the corresponding $\mathrm{T}_{2} / \mathrm{S}_{0}$ crossing point), whereas the slow component was assigned to the deactivation of the $T_{1}$ state (via the $T_{1} / S_{0}$ crossing point). These interpretations were based on the larger spin crossover regions computed for $T_{1}$ as compared to those of $T_{2}$, which could lead to more efficient excited-state trapping of the former state. On the other hand, the differences in the branching ratio of the two components was interpreted on the basis of the steeper MEP computed for the mer isomer of $\mathbf{C} \mathbf{1}$ (see Figure 12) with respect to that of the fac arrangement of C2 (see Figure 13).

This example, that constitutes to the best of our knowledge one of the first observation of such a subtle but yet crucial role of the isomerism in governing the excited-state lifetimes of $\mathrm{Fe}(\mathrm{II})$ complexes, is also paradigmatic in underlying the importance played by molecular modeling in unraveling the photophysics of such complexes and allowing for a coherent and global interpretation of time-resolved spectroscopy measurements in terms of state populations and PESs.

\section{Conclusions and perspectives}

The photophysics of iron complexes, and the still unresolved quest to obtain long-lived and luminescent $\mathrm{Fe}$ (II) complexes, is an urgent scientific challenge to be faced with possible huge outcomes and societal impact allowing to replace rare and highly toxic metals, such as ruthenium, in technological smart devices and biomedical applications.

It is also a domain in which modern excited-state molecular modeling can play a leading role in allowing a fair interpretation of complex experimental data, in suggesting original strategies to increase the excited-state lifetimes, and in providing some cheap pre-screening to select potential candidates to obtain luminescent or in general optical active compounds.

In most cases, some important information concerning the photophysical properties of iron complexes can be obtained by the simple positioning of the excited triplet and singlet adiabatic energy levels, e.g. assuring a differential stabilization of the ${ }^{3} \mathrm{MLCT}$ states with respect to ${ }^{3,5} \mathrm{MC}$ states. The scientific hypothesis underlying such a strategy is that the photophysics of iron compounds can be reverted to the one of ruthenium if the order between the ${ }^{3} \mathrm{MLCT}$ and ${ }^{3,5} \mathrm{MC}$ states is exchanged.

In this context, molecular modeling is also called to make a further step in the characterization of iron organometallic compounds and their photophysics. In particular, as we have underlined in the present review, the modeling of potential energy surfaces allows for a much better elucidation of the subtle photophysical processes that are at play in the deactivation processes. Relevant breakthroughs in the understanding of these photoresponses have been provided by the use of a wide variety of theoretical approaches that give access to more or less approximated potential energy surfaces at different computational costs. The choice of one or another depends mainly on the system under study and the desired level of accuracy and detail. For those cases in which a highly reliable description is needed, we recommend to use minimum energy path algorithms to obtain fully optimized and connected pathways between the considered geometries, combined with minimum-energy crossing points optimizations to describe the intersystem crossing processes.

An illustrative example is provided by the minimum energy path calculations on a recently synthesized bidentate $\mathrm{Fe}$ (II) complex, ${ }^{[57,198]}$ which have recently revealed unexpected influences of structural isomerism on the PESs, and have permitted to redefine some paradigm of the iron photophysics, such as the participation of the quintet states manifold, and to point to some original strategies to increase the lifetime. Indeed, and linked to the mentioned results, it has emerged that the deactivation pathway is invariably coupled to the enlargement of Fe-N bond distances, pointing such coordinate as the weak point leading to ${ }^{3} \mathrm{MLCT}$ deactivation. Hence, a rigidification of the octahedral coordination sphere could result in most beneficial effects on the excited state stabilization. In a first instance, such a flexibility decrease could proceed via the suppression of the Fe- 
$\mathrm{N}$ bonds. Indeed, in the impressive case reported by Wandmark and coworkers and leading to a ${ }^{3} \mathrm{MLCT}$ lifetime of 528 ps for an iron complex, all Fe-N connectivities have been replaced by $\mathrm{Fe}-$ carbene bonds. ${ }^{[55]}$ It is interesting to point out that also in the case of this complex, and as revealed by the X-ray structure of the MC triplet state, the deactivation proceeds via the enlargement of the Fe-carbene distance. Hence, a further rigidification could be necessary to provide even longer lifetime, in this respect one could envisage the possibility to achieve strongly caged and constrained coordination spheres, or to introduce extended $\pi$ stacked regions disfavoring the structural deformations coupled to the deactivation.

In the case of molecular modeling, the challenge will rely in precisely describe the subtle change brought to the ground and excited state PESs' topologies by such structural deformation and the effects on the possible photophysical pathways. In addition, and to better explore the degrees of freedom related to deactivation and the temporal evolution of the systems, static descriptions should be complemented by non-adiabatic molecular dynamics simulations to offer a one-to-one mapping between experimental and computational results, and to clearly identify the effects of the different normal modes in driving the overall photophysical processes.

Despite the important progress made so far, the route to stable and commercially available luminescent iron compounds appears to still be far ahead. However, it is also a task presenting many important scientific opportunities to challenge state-of-the-art computational methods and to unravel fundamental photophysical processes in a rich and still widely unexplored arena. Undoubtedly, iron photophysics has bright days to face in the near future.

\section{Acknowledgements}

The work is funded by the French Agence Nationale de la Recherche under the PhotIron project (ANR-16-CE07-0013-02). A.F.-M. acknowledges the French ANR for a postdoctoral contract, and the financial support from the project CTQ2017-87054-C2-2$\mathrm{P}$ of the Spanish Government.

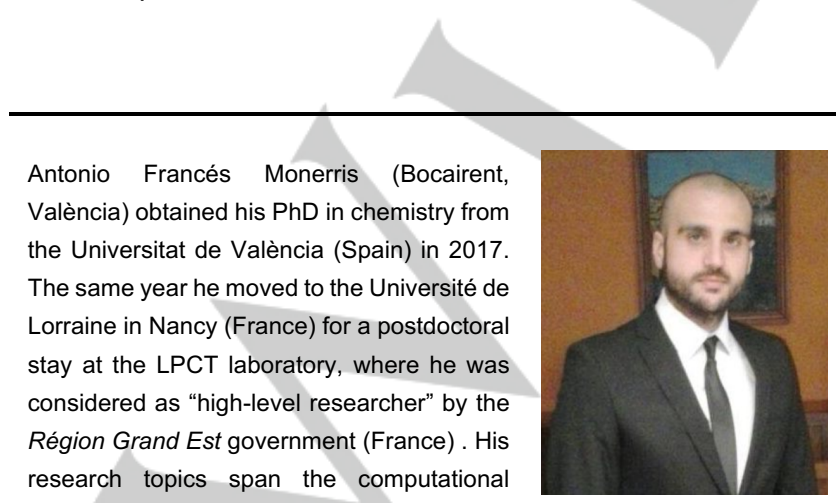

complexes and polyhedral borane hydrides, and the description of photoinduced phenomena in biological systems like nucleic acids.

Philippe C. Gros studied chemistry at the University of Lyon (France) and obtained his Ph.D in 1992. After two years as industry postdoctoral fellow, he entered the CNRS in 1994 and became Research Director in 2006. He is now first-class Research Director and Head of CNRS UMR L2CM in Nancy. His research interests include the design of new organometallic reagents for functionalization of heterocycles, transition metalcatalyzed cross-couplings for ligand synthesis, and the design of photo- and electroactive complexes for solar energy.

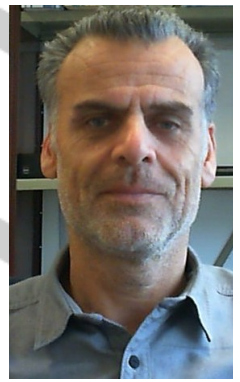

Xavier Assfeld is distinguished professor at the Université de Lorraine where he was hired in September 1995. He defended his Ph.D. the $13^{\text {th }}$ of July 1994 at the Université Henri Poincaré. He enjoyed a year in Minneapolis with Pr. Don Truhlar as a post-doc from September 1994 until September 1995. After serving more than ten years as the director of the graduate school of molecular physic and chemistry, he is since 2018 the head of the Department of Theoretical Physic and Theoretical Chemistry (LPCT). He was also the head of the French network of the CNRS from 2012 and the president of the physical chemistry division of of theoretical methods to study physical and chemical properties of complex molecular systems (proteins, DNA, solutions, ...). For example, he is at the origin of the LSCF QM/MM method.

Antonio Monari is associate professor (Maître de Conférence) at the Université de Lorraine, Nancy, since 2010. He obtained a Ph.D. in computational chemistry from the University of Bologna, Italy in 2007 and worked as a postdoc fellow in Bologna and in Toulouse Universities. After moving to Nancy his scientific interests are mostly focused on the elucidation of photochemical and photophysical processes in complex systems and complex environments. He is also strongly involved in the use of molecular modelling and simulation to interpret photobiological complexes phenomena such as DNA lesions

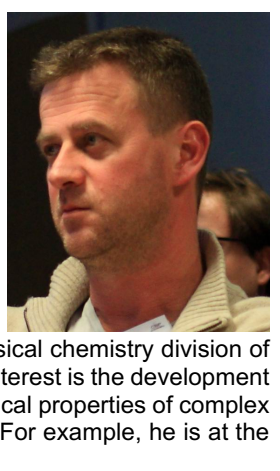
and their biolo

Mariachiara Pastore is researcher at the French National Centre for Scientific Research (CNRS) in the Laboratoire de Physique et chimie Théoriques, Université de Lorraine, Nancy, France. She was the recipient of the "Carla Roetti" prize 2014 from the Italian Chemistry Society (SCl), dedicated to theoretical and computational chemists under 40 and of the Ricercat@mente award in 2013 from the Italian National Research Council (CNR) for the Best Young Researcher under 35 years old in Chemical Science and

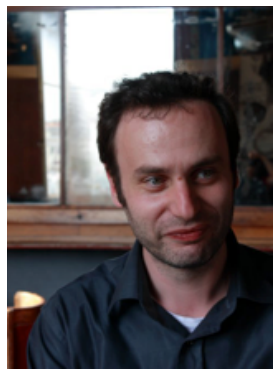
Materials Technology. Her research interests span from the development and application of highly correlated ab initio methods (multireference perturbation theories) and hybrid density functional theory/wavefunctionbased methods to the study of excited states in small and medium size molecules, to the characterization of the optical and charge separation properties in molecule/semiconductor interfaces. 
Keywords: iron complexes - Fe(II) complexes • photophysics • potential energy surface $\cdot$ excited states $\cdot$ molecular modelling $\bullet$ DFT $\cdot$ minimum energy path

[1] A. Listorti, J. Durrant, J. Barber, Nat. Mater. 2009, 8, 929930.

[2] R. J. Sension, Nature 2007, 446, 740-741.

[3] M. K. Brennaman, R. J. Dillon, L. Alibabaei, M. K. Gish, C. J. Dares, D. L. Ashford, R. L. House, G. J. Meyer, J. M. Papanikolas, T. J. Meyer, J. Am. Chem. Soc. 2016, 138, 13085-13102.

[4] K. E. Dalle, J. Warnan, J. J. Leung, B. Reuillard, S. Isabell, E. Reisner, Chem. Rev. 2019, 119, 2752-2875.

[5] M. K. Nazeeruddin, M. Grätzel, Struct. Bond. 2007, 123, 113-175.

[6] J. Zhao, S. Ji, W. Wu, W. Wu, H. Guo, J. Sun, H. Sun, Y. Liu, Q. Li, L. Huang, RSC Adv. 2012, 2, 1712-1728.

[7] C. A. Bignozzi, R. Argazzi, R. Boaretto, E. Busatto, S. Carli, F. Ronconi, S. Caramori, Coord. Chem. Rev. 2013, 257, 1472-1492.

[8] S. Aghazada, M. Nazeeruddin, Inorganics 2018, 6, 52.

[9] C. Mari, H. Huang, R. Rubbiani, M. Schulze, F. Würthner, C. Hui, G. Gasser, Eur. J. Inorg. Chem. 2016, 2017, 17451752.

[10] A. Zamora, G. Vigueras, V. Rodríguez, M. D. Santana, J. Ruiz, Coord. Chem. Rev. 2018, 360, 34-76.

[11] C. C. Konkankit, S. C. Marker, K. M. Knopf, J. J. Wilson, Dalt. Trans. 2018, 47, 9934-9974.

[12] G. A. Crosby, W. G. Perkins, D. M. Klassen, J. Chem. Phys. 1965, 43, 1498-1503.

[13] H. D. Gafney, A. W. Adamson, J. Am. Chem. Soc. 1972 94, 8238-8239.

[14] M. K. Nazeeruddin, C. Klein, P. Liska, M. Grätzel, in Coord. Chem. Rev., Elsevier, 2005, pp. 1460-1467.

[15] L. Han, A. Islam, H. Chen, C. Malapaka, B. Chiranjeevi, S. Zhang, X. Yang, M. Yanagida, Energy Environ. Sci. 2012, 5, 6057-6060.

[16] G. Koyyada, N. S. Pilli, J. H. Jung, K. K. Mandari, B. Shanigaram, M. Chandrasekharam, Int. J. Hydrogen Energy 2018, 43, 6963-6976.

[17] M. Pastore, F. De Angelis, J. Am. Chem. Soc. 2015, 137, 5798-5809.

[18] M. Pastore, A. Selloni, S. Fantacci, F. De Angelis, Springer, Berlin, Heidelberg, 2014, pp. 1-45.

[19] S. Yun, Y. Qin, A. R. Uhl, N. Vlachopoulos, M. Yin, D. Li, X. Han, A. Hagfeldt, Energy Environ. Sci. 2018, 11, 476-526.
[20] D. M. Arias-Rotondo, J. K. McCusker, Chem. Soc. Rev. 2016, 45, 5803-5820.

[21] S. Ardo, G. J. Meyer, Chem. Soc. Rev. 2009, 38, 115-164.

[22] A. Listorti, B. O’Regan, J. R. Durrant, Chem. Mater. 2011, 23, 3381-3399.

[23] F. Labat, I. Ciofini, H. P. Hratchian, M. J. Frisch, K. Raghavachari, C. Adamo, J. Phys. Chem. C 2011, 115, 4297-4306.

[24] N. Azzaroli, M. G. Lobello, A. Lapini, A. lagatti, L. Bussotti, M. Di Donato, G. Calogero, M. Pastore, F. De Angelis, P. Foggi, Phys. Chem. Chem. Phys. 2015, 17, 21594-21604.

[25] M. Chergui, Acc. Chem. Res. 2015, 48, 801-808.

[26] P. M. Kraus, M. Zürch, S. K. Cushing, D. M. Neumark, S. R. Leone, Nat. Rev. Chem. 2018, 2, 82-94.

[27] M. Chergui, Struct. Dyn. 2016, 3, 031001.

[28] A. Cannizzo, F. van Mourik, W. Gawelda, G. Zgrablic, C. Bressler, M. Chergui, Angew. Chemie Int. Ed. 2006, 45, 3174-3176.

[29] O. S. Wenger, J. Am. Chem. Soc. 2018, 140, 1352213533.

[30] J. E. Monat, J. K. McCusker, J. Am. Chem. Soc. 2000, 122, 4092-4097.

[31] W. Zhang, R. Alonso-Mori, U. Bergmann, C. Bressler, M. Chollet, A. Galler, W. Gawelda, R. G. Hadt, R. W. Hartsock, T. Kroll, et al., Nature 2014, 509, 345-348.

[32] M. Cammarata, R. Bertoni, M. Lorenc, H. Cailleau, S. Di Matteo, C. Mauriac, S. F. Matar, H. Lemke, M. Chollet, S. Ravy, et al., Phys. Rev. Lett. 2014, 113, 227402.

[33] G. Auböck, M. Chergui, Nat. Chem. 2015, 7, 629-633.

[34] D. Magde, J. K. McCusker, K. N. Walda, R. C. Dunn, J. D. Simon, D. N. Hendrickson, J. Am. Chem. Soc. 2005, 115, 298-307.

[35] A. Cannizzo, C. J. Milne, C. Consani, W. Gawelda, C. Bressler, F. van Mourik, M. Chergui, Coord. Chem. Rev. 2010, 254, 2677-2686.

[36] E. Jakubikova, D. N. Bowman, Acc. Chem. Res. 2015, 48, 1441-1449.

[37] S. Ferrere, B. A. Gregg, J. Am. Chem. Soc. 1998, 120, 843-844.

[38] G. J. Meyer, Inorg. Chem. 2005, 44, 6852-6864.

[39] J. K. McCusker, Science (80-. ). 2019, 363, 484-488.

[40] M. Kaupp, J. Comput. Chem. 2007, 28, 320-325.

[41] O. S. Wenger, Chem. - A Eur. J. 2019, DOI 10.1002/chem.201806148.

[42] J.-H. Shon, T. S. Teets, ACS Energy Lett. 2019, 4, 558- 
566.

[43] T. C. Motley, G. J. Meyer, NPG Asia Mater. 2016, 8, e261e261.

[44] T. C. B. Harlang, Y. Liu, O. Gordivska, L. A. Fredin, C. S. Ponseca, P. Huang, P. Chábera, K. S. Kjaer, H. Mateos, J. Uhlig, et al., Nat. Chem. 2015, 7, 883-889.

[45] F. N. Castellano, Nature 2017, 543, 627-628.

[46] L. L. Jamula, A. M. Brown, D. Guo, J. K. McCusker, Inorg. Chem. 2014, 53, 15-17.

[47] S. M. Fatur, S. G. Shepard, R. F. Higgins, M. P. Shores, N. H. Damrauer, J. Am. Chem. Soc. 2017, 139, 4493-4505.

[48] D. N. Bowman, A. Bondarev, S. Mukherjee, E. Jakubikova, Inorg. Chem. 2015, 54, 8786-8793.

[49] P. Zimmer, L. Burkhardt, A. Friedrich, J. Steube, A. Neuba, R. Schepper, P. Müller, U. Flörke, M. Huber, S. Lochbrunner, et al., Inorg. Chem. 2017, 57, 360-373.

[50] T. Duchanois, T. Etienne, M. Beley, X. Assfeld, E. A. Perpète, A. Monari, P. C. Gros, Eur. J. Inorg. Chem. 2014, 2014, 3747-3753.

[51] I. M. Dixon, F. Alary, M. Boggio-Pasqua, J. L. Heully, Dalt. Trans. 2015, 44, 13498-13503.

[52] L. Liu, T. Duchanois, T. Etienne, A. Monari, M. Beley, X. Assfeld, S. Haacke, P. C. Gros, Phys. Chem. Chem. Phys. 2016, 18, 12550-12556.

[53] T. Duchanois, T. Etienne, C. Cebrián, L. Liu, A. Monari, M. Beley, X. Assfeld, S. Haacke, P. C. Gros, Eur. J. Inorg. Chem. 2015, 2015, DOI 10.1002/ejic.201500142.

[54] M. Pastore, T. Duchanois, L. Liu, A. Monari, X. Assfeld, S. Haacke, P. C. Gros, Phys. Chem. Chem. Phys. 2016, 18, 28069-28081.

[55] P. Chábera, K. S. Kjaer, O. Prakash, A. Honarfar, Y. Liu, L. A. Fredin, T. C. B. Harlang, S. Lidin, J. Uhlig, V. Sundström, et al., J. Phys. Chem. Lett. 2018, 9, 459-463.

[56] P. Chábera, Y. Liu, O. Prakash, E. Thyrhaug, A. El Nahhas, A. Honarfar, S. Essén, L. A. Fredin, T. C. B. Harlang, K. S. Kjær, et al., Nature 2017, 543, 695-699.

[57] A. Francés-Monerris, K. Magra, M. Darari, C. Cebrián, M. Beley, E. Domenichini, S. Haacke, M. Pastore, X. Assfeld, P. C. Gros, et al., Inorg. Chem. 2018, 57, 10431-10441.

[58] S. Mukherjee, D. N. Bowman, E. Jakubikova, Inorg. Chem. 2015, 54, 560-569.

[59] K. S. Kjær, N. Kaul, O. Prakash, P. Chábera, N. W. Rosemann, A. Honarfar, O. Gordivska, L. A. Fredin, K. E. Bergquist, L. Häggström, et al., Science (80-. ). 2019, 363, 249-253.
J. Am. Chem. Soc. 2016, 138, 2949-2952.

[61] Y. Liu, T. Harlang, S. E. Canton, P. Chábera, K. SuárezAlcántara, A. Fleckhaus, D. A. Vithanage, E. Göransson, A. Corani, R. Lomoth, et al., Chem. Commun. 2013, 49, 6412.

[62] Y. Liu, P. Persson, V. Sundström, K. Wärnmark, Acc. Chem. Res. 2016, 49, 1477-1485.

[63] T. Duchanois, L. Liu, M. Pastore, A. Monari, C. Cebrián, Y. Trolez, M. Darari, K. Magra, A. Francés-Monerris, E. Domenichini, et al., Inorganics 2018, 6, 63.

[64] T. Duchanois, T. Etienne, C. Cebrián, L. Liu, A. Monari, M. Beley, X. Assfeld, S. Haacke, P. C. Gros, Eur. J. Inorg. Chem. 2015, 2015, 2469-2477.

[65] I. M. Dixon, G. Boissard, H. Whyte, F. Alary, J. L. Heully, Inorg. Chem. 2016, 55, 5089-5091.

[66] P. Stock, D. Wiedemann, H. Petzold, G. Hörner, Inorganics 2017, 5, 60 .

[67] G. Grell, N. Engel, S. I. Bokarev, A. Moguilevski, S. G. Aziz, E. F. Aziz, I. Y. Kiyan, M. Wilke, A. A. Raheem, O. Kühn, ChemPhysChem 2016, 18, 465-469.

[68] C. Sousa, M. Llunell, A. Domingo, C. De Graaf, Phys. Chem. Chem. Phys. 2018, 20, 2351-2355.

[69] L. A. Fredin, M. Pápai, E. Rozsályi, G. Vankó, K. Wärnmark, V. Sundström, P. Persson, J. Phys. Chem. Lett. 2014, 5, 2066-2071.

[70] M. Pápai, T. J. Penfold, K. B. Møller, J. Phys. Chem. C 2016, 120, 17234-17241.

[71] M. Pápai, M. Abedi, G. Levi, E. Biasin, M. M. Nielsen, K. B. Møller, J. Phys. Chem. C 2019, 123, 2056-2065.

[72] D. Leshchev, T. C. B. Harlang, L. A. Fredin, D. Khakhulin, Y. Liu, E. Biasin, M. G. Laursen, G. E. Newby, K. Haldrup, M. M. Nielsen, et al., Chem. Sci. 2018, 9, 405-414.

[73] D. C. Ashley, E. Jakubikova, Coord. Chem. Rev. 2017, 337, 97-111.

[74] J. Nance, D. N. Bowman, S. Mukherjee, C. T. Kelley, E. Jakubikova, Inorg. Chem. 2015, 54, 11259-11268.

[75] C. Sousa, M. Alías, A. Domingo, C. de Graaf, Chem. - A Eur. J. 2019, 25, 1152-1164.

[76] D. Escudero, Springer, Cham, 2019, pp. 259-287.

[77] C. Amovilli, V. Barone, R. Cammi, E. Cancès, M. Cossi, B. Mennucci, C. S. Pomelli, J. Tomasi, Adv. Quantum Chem. 1998, 32, 227-261.

[78] B. Mennucci, Wiley Interdiscip. Rev. Comput. Mol. Sci. 2012, 2, 386-404.

[79] K. Boguslawski, C. R. Jacob, M. Reiher, J. Chem. Theory Comput. 2011, 7, 2740-2752. 
[80] S. Song, M. C. Kim, E. Sim, A. Benali, O. Heinonen, K. Burke, J. Chem. Theory Comput. 2018, 14, 2304-2311.

[81] D. N. Bowman, E. Jakubikova, Inorg. Chem. 2012, 51, 6011-6019.

[82] M. Reiher, Inorg. Chem. 2002, 41, 6928-6935.

[83] L. Wilbraham, C. Adamo, I. Ciofini, J. Chem. Phys. 2018, 148, 041103.

[84] M. Swart, A. R. Groenhof, A. W. Ehlers, K. Lammertsma, J. Phys. Chem. A 2004, 108, 5479-5483.

[85] G. Ganzenmüller, N. Berkaïne, A. Fouqueau, M. E. Casida, M. Reiher, J. Chem. Phys. 2005, 122, 234321.

[86] E. I. Ioannidis, H. J. Kulik, J. Phys. Chem. A 2017, 121, 874-884.

[87] A. Fouqueau, S. Mer, M. E. Casida, L. M. L. Daku, A. Hauser, T. Mineva, F. Neese, J. Chem. Phys. 2004, 120, 9473-9486.

[88] L. M. Lawson Daku, A. Vargas, A. Hauser, A. Fouqueau, M. E. Casida, ChemPhysChem 2005, 6, 1393-1410.

[89] F. Neese, Coord. Chem. Rev. 2009, 253, 526-563.

[90] M. Swart, J. Chem. Theory Comput. 2008, 4, 2057-2066.

[91] S. Ye, F. Neese, Inorg. Chem. 2010, 49, 772-774.

[92] K. P. Kepp, Coord. Chem. Rev. 2012, 257, 196-209.

[93] S. Zein, S. A. Borshch, P. Fleurat-Lessard, M. E. Casida, H. Chermette, J. Chem. Phys. 2007, 126, 014105

[94] L. Petit, P. Maldivi, C. Adamo, J. Chem. Theory Comput. 2005, 1, 953-962.

[95] C. J. Cramer, D. G. Truhlar, Phys. Chem. Chem. Phys. 2009, 11, 10757-10816.

[96] C. Daniel, Coord. Chem. Rev. 2003, 238-239, 143-166.

[97] S. M. Tekarli, M. L. Drummond, T. G. Williams, T. R. Cundari, A. K. Wilson, J. Phys. Chem. A 2009, 113, 86078614.

[98] A. Rosa, G. Ricciardi, O. Gritsenko, E. J. Baerends, Springer, Berlin, Heidelberg, 2011, pp. 49-116.

[99] A. Vlček, S. Záliš, Coord. Chem. Rev. 2007, 251, 258-287.

[100] C. Latouche, D. Skouteris, F. Palazzetti, V. Barone, J. Chem. Theory Comput. 2015, 11, 3281-3289.

[101] A. J. Atkins, F. Talotta, L. Freitag, M. Boggio-Pasqua, L. González, J. Chem. Theory Comput. 2017, 13, 4123-4145.

[102] S. Fantacci, F. De Angelis, Coord. Chem. Rev. 2011, 255, 2704-2726.

[103] A. Selloni, M. Grätzel, S. Ito, G. Viscardi, F. De Angelis, P. Liska, M. K. Nazeeruddin, S. Fantacci, B. Takeru, J. Am. Chem. Soc. 2005, 127, 16835-16847.
[104] S. I. Bokarev, O. S. Bokareva, O. Kühn, Coord. Chem. Rev. 2015, 304-305, 133-145.

[105] K. Pierloot, S. Vancoillie, J. Chem. Phys. 2006, 125, 124303.

[106] A. Rudavskyi, C. Sousa, C. De Graaf, R. W. A. Havenith, R. Broer, J. Chem. Phys. 2014, 140, 184318.

[107] K. Pierloot, S. Vancoillie, J. Chem. Phys. 2008, 128, 034104.

[108] L. M. Lawson Daku, F. Aquilante, T. W. Robinson, A. Hauser, J. Chem. Theory Comput. 2012, 8, 4216-4231.

[109] J. Cirera, M. Via-Nadal, E. Ruiz, Inorg. Chem. 2018, 57, 14097-14105.

[110] L. Wilbraham, P. Verma, D. G. Truhlar, L. Gagliardi, I. Ciofini, J. Phys. Chem. Lett. 2017, 8, 2026-2030.

[111] K. Andersson, P. A. Malmqvist, B. O. Roos, J. Chem. Phys. 1992, 96, 1218-1226.

[112] C. Angeli, M. Pastore, R. Cimiraglia, Theor. Chem. Acc. 2007, 117, 743-754.

[113] L. González, D. Escudero, L. Serrano-Andrés, ChemPhysChem 2012, 13, 28-51.

[114] A. Domingo, M. À. Carvajal, C. de Graaf, K. Sivalingam, F. Neese, C. Angeli, Theor. Chem. Acc. 2012, 131, 1-13.

[115] M. Pastore, F. De Angelis, C. Angeli, Theor. Chem. Acc. 2016, 135, 108.

[116] Q. M. Phung, S. Wouters, K. Pierloot, J. Chem. Theory Comput. 2016, 12, 4352-4361.

[117] D. Escudero, L. González, J. Chem. Theory Comput. 2012, 8, 203-213.

[118] A. Delgado, S. Corni, G. Goldoni, Theor. Chem. Acc. 2012, 131, 1-14.

[119] M. Pápai, G. Vankó, C. De Graaf, T. Rozgonyi, J. Chem. Theory Comput. 2013, 9, 509-519.

[120] K. Pierloot, Int. J. Quantum Chem. 2011, 111, 3291-3301.

[121] N. Ben Amor, S. Záliš, C. Daniel, Int. J. Quantum Chem. 2006, 106, 2458-2469.

[122] A. L. B. Formiga, S. Vancoillie, K. Pierloot, Inorg. Chem. 2013, 52, 10653-10663.

[123] K. Pierloot, Q. M. Phung, A. Domingo, J. Chem. Theory Comput. 2017, 13, 537-553.

[124] Q. M. Phung, M. Feldt, J. N. Harvey, K. Pierloot, J. Chem. Theory Comput. 2018, 14, 2446-2455.

[125] S. K. Singh, M. Atanasov, F. Neese, J. Chem. Theory Comput. 2018, 14, 4662-4677.

[126] M. Radoń, Phys. Chem. Chem. Phys. 2019, 21, 48544870. 
[127] S. Vancoillie, H. Zhao, V. T. Tran, M. F. A. Hendrickx, K. Pierloot, J. Chem. Theory Comput. 2011, 7, 3961-3977.

[128] M. Pastore, C. Angeli, R. Cimiraglia, Chem. Phys. Lett. 2006, 422, 522-528.

[129] N. S. Hush, J. R. Reimers, Chem. Rev. 2000, 100, 775786.

[130] T. Véry, D. Ambrosek, M. Otsuka, C. Gourlaouen, X. Assfeld, A. Monari, C. Daniel, Chem. - A Eur. J. 2014, 20, 12901-12909.

[131] P. Chen, T. J. Meyer, Chem. Rev. 2002, 98, 1439-1478.

[132] A. Rondi, Y. Rodriguez, T. Feurer, A. Cannizzo, Acc. Chem. Res. 2015, 48, 1432-1440.

[133] M. E. Moret, I. Tavernelli, M. Chergui, U. Rothlisberger, Chem. - A Eur. J. 2010, 16, 5889-5894.

[134] K. S. Kjær, W. Zhang, R. Alonso-Mori, U. Bergmann, M. Chollet, R. G. Hadt, R. W. Hartsock, T. Harlang, T. Kroll, K. Kubiček, et al., Struct. Dyn. 2017, 4, 044030.

[135] S. Piccinin, D. Rocca, M. Pastore, J. Phys. Chem. C 2017, 121, 22286-22294

[136] G. Prampolini, F. Ingrosso, A. Segalina, S. Caramori, P. Foggi, M. Pastore, J. Chem. Theory Comput. 2019, 15, 529-545.

[137] M. E. Moret, I. Tavernelli, U. Rothlisberger, J. Phys. Chem. B 2009, 113, 7737-7744.

[138] A. Chantzis, T. Very, A. Monari, X. Assfeld, J. Chem Theory Comput. 2012, 8, 1536-1541.

[139] E. Zvereva, J. Segarra-Martí, M. Marazzi, J. Brazard, A Nenov, O. Weingart, J. Léonard, M. Garavelli, I. Rivalta, E. Dumont, et al., Photochem. Photobiol. Sci. 2018, 17, 323331.

[140] F. Santoro, R. Improta, A. Lami, J. Bloino, V. Barone, J. Chem. Phys. 2007, 126, 084509.

[141] V. Barone, J. Bloino, M. Biczysko, F. Santoro, J. Chem. Theory Comput. 2009, 5, 540-554.

[142] I. M. Dixon, G. Boissard, H. Whyte, F. Alary, J. L. Heully, Inorg. Chem. 2016, 55, 5089-5091.

[143] J. Sanz García, M. Boggio-Pasqua, I. Ciofini, M. Campetella, J. Comput. Chem. 2019, 0, DOI $10.1002 /$ jcc. 25800

[144] W. Zhang, K. S. Kjær, R. Alonso-Mori, U. Bergmann, M. Chollet, L. A. Fredin, R. G. Hadt, R. W. Hartsock, T. Harlang, T. Kroll, et al., Chem. Sci. 2017, 8, 515-523.

[145] L. A. Fredin, K. Wärnmark, V. Sundström, P. Persson, ChemSusChem 2016, 9, 667-675.

[146] Y. Liu, K. S. Kjær, L. A. Fredin, P. Chábera, T. Harlang, S. E. Canton, S. Lidin, J. Zhang, R. Lomoth, K. Bergquist, et al., Chem. - A Eur. J. 2014, 21, 3628-3639.

[147] B. Ordejón, C. de Graaf, C. Sousa, J. Am. Chem. Soc. 2008, 130, 13961-13968.

[148] C. Sousa, C. De Graaf, A. Rudavskyi, R. Broer, J. Tatchen, M. Etinski, C. M. Marian, Chem. - A Eur. J. 2013, 19, 17541-17551.

[149] N. Suaud, M.-L. Bonnet, C. Boilleau, P. Labèguerie, N. Guihéry, J. Am. Chem. Soc. 2009, 131, 715-722.

[150] C. De Graaf, C. Sousa, Int. J. Quantum Chem. 2011, 111, 3385-3393.

[151] G. Vankó, A. Bordage, M. Pápai, K. Haldrup, P. Glatzel, A. M. March, G. Doumy, A. Britz, A. Galler, T. Assefa, et al., J. Phys. Chem. C 2015, 119, 5888-5902.

[152] D. C. Ashley, E. Jakubikova, Inorg. Chem. 2018, 57, 55855596.

[153] M. Pápai, G. Vankó, T. Rozgonyi, T. J. Penfold, J. Phys. Chem. Lett. 2016, 7, 2009-2014.

[154] J. Nance, E. Jakubikova, C. T. Kelley, J. Chem. Theory Comput. 2014, 10, 2942-2949.

[155] D. Sheppard, R. Terrell, G. Henkelman, J. Chem. Phys. 2008, 128, 134106.

[156] M. A. Robb, F. Bernardi, M. Olivucci, Pure Appl. Chem. 1995, 67, 783-789.

[157] D. Roca-Sanjuán, F. Aquilante, R. Lindh, Wiley Interdiscip. Rev. Comput. Mol. Sci. 2012, 2, 585-603.

[158] M. (Massimo) Olivucci, Computational Photochemistry, Elsevier, 2005

[159] M. A. Robb, M. Garavelli, M. Olivucci, F. Bernardi, John Wiley \& Sons, Ltd, 2007, pp. 87-146.

[160] M. G. S. Londesborough, J. Dolanský, T. Jelínek, J. D. Kennedy, I. Císařová, R. D. Kennedy, D. Roca-Sanjuán, A. Francés-Monerris, K. Lang, W. Clegg, Dalt. Trans. 2018, 47, 1709-1725.

[161] L. A. Estrada, A. Francés-Monerris, I. Schapiro, M. Olivucci, D. Roca-Sanjuán, Phys. Chem. Chem. Phys. 2016, 18, 32786-32795.

[162] L. Serrano-Andres, M. Merchan, A. C. Borin, Proc. Natl. Acad. Sci. 2006, 103, 8691-8696.

[163] F. Liu, Y. Liu, L. De Vico, R. Lindh, J. Am. Chem. Soc. 2009, 131, 6181-6188.

[164] J. Segarra-Martí, A. Francés-Monerris, D. Roca-Sanjuán, M. Merchán, J. Segarra-Martí, A. Francés-Monerris, D. Roca-Sanjuán, M. Merchán, Molecules 2016, 21, 1666.

[165] J. Conyard, I. A. Heisler, Y. Chan, P. C. Bulman Page, S. R. Meech, L. Blancafort, Chem. Sci. 2018, 9, 1803-1812. 
[166] A. Frances-Monerris, M. Merchan, D. Roca-Sanjuan, J. Phys. Chem. B 2014, 118, 2932-2939.

[167] M. E. Martin, F. Negri, M. Olivucci, J. Am. Chem. Soc. 2004, 126, 5452-5464.

[168] L. A. Estrada, A. Frances-Monerris, I. Schapiro, M. Olivucci, D. Roca-Sanjuan, Phys. Chem. Chem. Phys. 2016, 18, 32786-32795.

[169] A. Cembran, F. Bernardi, M. Olivucci, M. Garavelli, Proc. Natl. Acad. Sci. U. S. A. 2005, 102, 6255 LP - 6260.

[170] G. N. Simm, A. C. Vaucher, M. Reiher, J. Phys. Chem. A 2019, 123, 385-399.

[171] H. B. Schlegel, Wiley Interdiscip. Rev. Comput. Mol. Sci. 2011, 1, 790-809.

[172] S. Maeda, Y. Harabuchi, Y. Ono, T. Taketsugu, K. Morokuma, Int. J. Quantum Chem. 2015, 115, 258-269.

[173] M. J. Frisch, G. W. Trucks, H. B. Schlegel, G. E. Scuseria, M. A. Robb, J. R. Cheeseman, G. Scalmani, V. Barone, B. Mennucci, G. A. Petersson, et al., Revis. D.01 2010, Gaussian Inc., Wallingford CT.

[174] F. Aquilante, J. Autschbach, R. K. Carlson, L. F. Chibotaru, M. G. Delcey, L. De Vico, I. Fdez. Galván, N. Ferré, L. M. Frutos, L. Gagliardi, et al., J. Comput. Chem. 2016, 37, 506-541.

[175] F. Neese, Wiley Interdiscip. Rev. Comput. Mol. Sci. 2012, 2, 73-78.

[176] TURBOMOLE V7.1 2016, "TURBOMOLE: Program Package for ab initio Electronic Structure Calculations," can be found under http://www.turbomole.com/, 2016.

[177] G. te Velde, F. M. Bickelhaupt, E. J. Baerends, C. Fonseca Guerra, S. J. A. van Gisbergen, J. G. Snijders, T. Ziegler, J. Comput. Chem. 2001, 22, 931-967.

[178] Y. Shao, Z. Gan, E. Epifanovsky, A. T. B. Gilbert, M. Wormit, J. Kussmann, A. W. Lange, A. Behn, J. Deng, X. Feng, et al., Mol. Phys. 2015, 113, 184-215.

[179] M. W. Schmidt, K. K. Baldridge, J. A. Boatz, S. T. Elbert, M. S. Gordon, J. H. Jensen, S. Koseki, N. Matsunaga, K. A. Nguyen, S. Su, et al., J. Comput. Chem. 1993, 14, 13471363.

[180] J. N. Harvey, Phys. Chem. Chem. Phys. 2007, 9, 331-343.

[181] M. J. Bearpark, M. A. Robb, H. Bernhard Schlegel, Chem.
Phys. Lett. 1994, 223, 269-274.

[182] J. D. Snyder, D. H. Ess, 2017.

[183] I. M. Dixon, F. Alary, M. Boggio-Pasqua, J.-L. Heully, Inorg. Chem. 2013, 52, 13369-13374.

[184] T. J. Penfold, E. Gindensperger, C. Daniel, C. M. Marian, Chem. Rev. 2018, 118, 6975-7025.

[185] K. Aidas, C. Angeli, K. L. Bak, V. Bakken, R. Bast, L. Boman, O. Christiansen, R. Cimiraglia, S. Coriani, P. Dahle, et al., Wiley Interdiscip. Rev. Comput. Mol. Sci. 2014, 4, 269-284.

[186] J. N. Harvey, Wiley Interdiscip. Rev. Comput. Mol. Sci. 2014, 4, 1-14.

[187] A. O. Lykhin, D. S. Kaliakin, G. E. Depolo, A. A. Kuzubov, S. A. Varganov, Int. J. Quantum Chem. 2016, 116, 750761.

[188] D. Wales, Energy Landscapes: Applications to Clusters, Biomolecules and Glasses, Cambridge University Press, 2003.

[189] I. S. Ufimtsev, T. J. Martinez, J. Chem. Theory Comput. 2009, 5, 2619-2628.

[190] T. Etienne, X. Assfeld, A. Monari, J. Chem. Theory Comput. 2014, 10, 3896-3905.

[191] T. Etienne, J. Chem. Theory Comput. 2015, 11, 16921699.

[192] M. Kasha, Discuss. Faraday Soc. 1950, 9, 14-19.

[193] I. M. Dixon, F. Alary, M. Boggio-Pasqua, J. L. Heully, Inorg. Chem. 2013, 52, 13369-13374.

[194] T. Harlang, K.-E. Bergquist, J. Zhang, K. Wärnmark, K. S. Kjaer, R. Lomoth, Y. Liu, S. Lidin, P. Chábera, L. A. Fredin, et al., Chem. - A Eur. J. 2014, 21, 3628-3639.

[195] S. Arroliga-Rocha, D. Escudero, Inorg. Chem. 2018, 57, 12106-12112.

[196] A. Soupart, F. Alary, J. L. Heully, P. I. P. Elliott, I. M. Dixon, Inorg. Chem. 2018, 57, 3192-3196.

[197] D. Escudero, ChemPhotoChem 2019, cptc.201900029.

[198] K. Magra, E. Domenichini, A. Francés-Monerris, C. Cebrian, M. Beley, M. Darari, M. Pastore, A. Monari, X. Assfeld, S. Haacke, et al., Inorg. Chem. 2019, accepted. 


\section{Entry for the Table of Contents}

\section{MINIREVIEW}

This work provides an overview of the different approaches used to compute the potential energy surfaces of $\mathrm{Fe}$ (II) complexes.

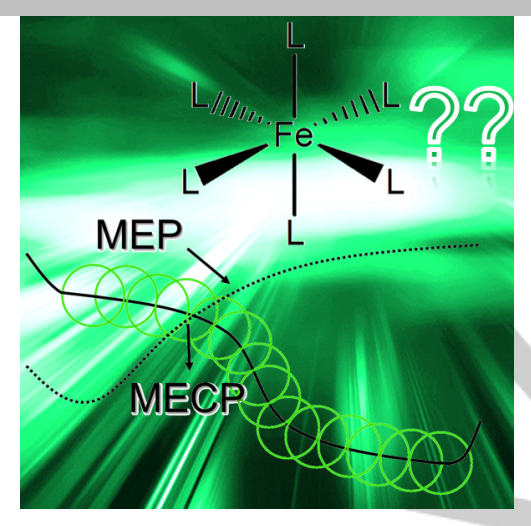

Antonio Francés-Monerris, ${ }^{*}$ Philippe $C$. Gros, Xavier Assfeld, Antonio Monari, and Mariachiara Pastore*

Page No. - Page No.

Toward luminescent iron complexes: unraveling the photophysics by computing potential energy surfaces 